\title{
Der Stuttgarter Baumeister Georg Stegle (ca. 1548-1598) und Schloss Weikersheim
}

\author{
von Jost Weyer
}

Georg Stegle gehört zusammen mit Georg Beer, Blasius Berwart und Elias Gunzenhäuser zu den bekannteren Baumeistern am Hof Herzog Ludwigs von Württemberg in Stuttgart, zu denen als berühmtester Heinrich Schickhardt zählt, der unter Ludwigs Nachfolger Friedrich der maßgebende Baumeister wurde. Über Stegles Leben und Werk gibt es an verschiedenen Stellen Informationen, wobei als wichtigste Quellen das „Neue Württembergische Dienerbuch“" und die „Renaissance im Herzogtum Württemberg“ von Fleischhauer ${ }^{2}$ zu nennen sind. Hier sollen die Einzelinformationen zu einem wohl immer noch lückenhaften Gesamtbild zusammengefasst werden.

Ein zweiter Gesichtspunkt betrifft die Bedeutung, die Stegle für die Konzipierung des Baus von Schloss Weikersheim hatte. Obwohl von einem Autor gründlich recherchiert, wurden die zutreffenden Ergebnisse von einigen anderen Autoren ignoriert und Stegles Verdienste anderen Baumeistern zugesprochen. Diese Fehldeutungen haben auch Eingang in den offiziellen Schlossführer von Schloss Weikersheim gefunden. Entdeckungen aus neuester Zeit sollen den wahren Sachverhalt klären.

\section{Stegle im Dienst der Herzöge von Württemberg}

Georg Stegle (auch Steglin) wurde um 1548 in Drackenstein (Schwäbische Alb, heute Kreis Göppingen) geboren. Er wird von 1564 bis 1596 als Kammerdiener bezeichnet, stand also schon zur Zeit von Herzog Christoph (1515-1568, reg. seit 1550) im württembergischen Dienst. 1581 wird er zum ersten Mal Baumeister genannt. 1584, 1593 und 1597/98 fungierte er als Bau- oder Holzverwalter. Die längste Zeit seiner beruflichen Tätigkeit fällt in die Regierungszeit von Herzog Ludwig von Württemberg (1554-1593, reg. seit 1568). Stegle heiratete 1575 oder 1576. 1598 starb er in Stuttgart. ${ }^{3}$

1 Walther Pfeilsticker: Neues Württembergisches Dienerbuch. Bd. 1. Stuttgart 1957, § 278 und 1944.

2 Werner Fleischhauer: Renaissance im Herzogtum Württemberg. Stuttgart [1971], S. 80 ff. (dort fälschlich Jakob Stegle).

3 Pfeilsticker (wie Anm. 1). 
Als Herzog Ludwig regierte, war sein Vetter Friedrich (1557-1608) Graf von Mömpelgard, der dann nach Ludwigs Tod 1593 als Herzog Friedrich I. von Württemberg sein Nachfolger wurde. Friedrich war ein baulustiger Herrscher, hatte aber als Graf nicht genügend finanzielle Mittel zur Verfügung. Im Dezember 1581 schrieb er an Ludwig, dass er Stegles Dienste für seine Bauten in Mömpelgard benötige, da dieser ,derselben Gelegenheit nunmehr allein Bericht und Erfahrung angenommen hat". Erst auf langes Bitten schickte er Stegle im Juni und Juli 1582 nach Mömpelgard, da er bei ihm an einem großen Werk zu arbeiten habe.

Ludwig hatte für Friedrichs Aktivitäten auf architektonischem Gebiet wenig Verständnis und beurlaubte Stegle und seine anderen Baumeister nur für kurze Zeit und konkrete Vorhaben, deren Dringlichkeit Friedrich nachweisen musste. In den Jahren 1583 und 1585 wurde Stegle noch einmal nach Mömpelgard beurlaubt, dann aber nicht mehr, trotz weiterer Bitten des Grafen. Die schnelle Beurlaubung Anfang $1585 \mathrm{kam}$ dadurch zustande, dass unterhalb von Schloss Mömpelgard die herrschaftliche Scheune einschließlich der Futtervorräte abgebrannt war. Welche Tätigkeiten Stegle im Detail in Mömpelgard ausführte, geht aus den Akten nicht hervor, ebenso wenig, an welchen Bauvorhaben er unter Ludwig beteiligt war. Man weiß nur aus anderen Quellen, dass er 1574 für Herzog Wilhelm V. von Bayern einen Abriss des Stuttgarter Lustgartens machte und 1588 auf dem Hohenasperg zu tun hatte. ${ }^{4}$

Im Jahr 1593 starb Herzog Ludwig, und nun standen seinem Vetter Friedrich als Herzog wesentlich größere Geldbeträge zur Realisierung seiner Bauvorhaben zur Verfügung. Im Sommer 1596 schickte Friedrich seinen Leibarzt, den schweizerischen Arzt und Botaniker Jean Bauhin, nach Boll in der Nähe von Göppingen, um über die Heilkraft der dortigen Quellen ein fachmännisches Urteil abzugeben. Friedrich hatte kurz vorher diese Quellen fassen lassen und Schickhardt mit der Errichtung eines Badhauses und der Anlage eines Parks beauftragt, was zwischen 1595 und 1597 geschah. Die Gestaltung des Parks von Bad Boll lag in den Händen von Stegle, unterstützt von zwei Gärtnern aus Göppingen und Stuttgart und zahlreichen Hilfskräften, beraten von Bauhin. Es wurde ein Lustgarten im Stil der Renaissance, wobei sich unter den Zierpflanzen auch die aus Amerika eingeführten Tabak- und Kartoffelstauden befanden. ${ }^{5}$

\section{Die „Anhaltische Heimführung“}

Im Jahr 1571 heirateten Ludwigs Schwester Eleonora, geborene Herzogin von Württemberg, und Joachim Ernst, Fürst von Anhalt. Die Hochzeit wurde Anfang

4 Fleischhauer (wie Anm. 2), S. 73, 80 ff. Paul Sauer: Herzog Friedrich I. von Württemberg $1557-$ 1608. Ungestümer Reformer und weltgewandter Autokrat. München 2003, S. 69, 86 f.

5 Fleischhauer (wie Anm. 2), S. 315; Sauer (wie Anm. 4), S. 229 f. 
Mai in Dessau gefeiert, wohin die Braut von Stuttgart über zahlreiche Zwischenstationen geleitet wurde. Sie wurde begleitet von Herzogin Anna Maria von Württemberg, Gemahlin des 1568 verstorbenen Herzogs Christoph und Mutter von Eleonora und Ludwig, und von ihrem Bruder Ludwig, der damals noch unverheiratet war. In ihrem Gefolge befand sich auch Georg Stegle, der damals etwa dreiundzwanzig Jahre alt war. Er verfasste ein ausführliches Reisetagebuch mit dem Kurztitel „Anhaltische Heimführung“, in dem alle Stationen auf der Hin- und Rückreise beschrieben sind. ${ }^{6}$ Den Bericht widmete er dem Bräutigam, Joachim Ernst von Anhalt. Gegenüber dem Titelblatt ist zu lesen: Euer Fürstlichen Gnaden unndertheniger, williger Georg Stegle, Furstlicher Wurtembergischer Diener.

Stegle war für alles interessiert, was ihm auf der Reise begegnete, berichtete über die Städte mit ihren Bauwerken und natürlich auch ausführlich über die Hochzeitsfeierlichkeiten. Besondere Aufmerksamkeit widmete er den Schlössern und den damit zusammenhängenden Gartenanlagen - den „Lustgärten“ und „Lusthäusern“. Ausführlich beschreibt er die Schlösser in Kassel, Weimar und Arnstadt.

Vom Schloss in Kassel sagt er: Dis Schloß ist gar zierlich in die Viereckh gebauet, mit einer Wendeltreppe in jeder Ecke. Unten im Schloss befanden sich die Wirtschaftsräume, mitten im Hof stand ein Röhrenbrunnen. Im Schloss gab es unter anderem einen langen, gewölbten Saal, 181 Schuh lang, 38 Schuh breit, einen zweiten großen Saal zum Empfang hochgestellter Persönlichkeiten, ein fürstliches Gemach für den Landgrafen Wilhelm von Hessen mit einem großen Wandbild eines Elefanten, vil herliche Furstengemach sambt dem Frauenzimmer, welche mit schönem Mahlwerckh und künstlicher Schreinerarbait geziert seindt, und zwei besonders schöne, von ihm ausführlich beschriebene Gemächer. Er erwähnt auch das Schließsystem mit einem Schlüssel für jede Tür und einem Hauptschlüssel. Etwas entfernt vom Schloss, getrennt durch die Fulda, lag ein Lustgarten mit einem Lusthaus. Der Garten war lustig gebauet mit welschen Compartamenta, in denen sich viele schöne Pflanzen und wohlriechende Kräuter befanden. Das viereckige, von einer Altane umgebene Lusthaus enthielt oben einen Saal, dessen Wände und Decke ausgemalt waren. ${ }^{7}$

Das Schloss in Weimar hatte einen großen Hof mit einem Röhrenbrunnen. Im Schloss befanden sich große Säle und Fürstengemächer. Die schöne Hofkapelle war mit zwei Emporen übereinander und einer stattlichen Orgel versehen. An das Schloss grenzte ein Lustgarten, durch den die Ilmenau floss, mit Obstbäumen und schönen Pflanzen. In diesem Garten befand sich ein gewaltigs Lusthaus mit einem viereckheten Garten, alles uff die französische Manier. In dem von

6 Georg Stegle: Herrn Joachim Ernsten Fürsten zu Anhalt mit Herzogin Eleonoren, geboren zu Württemberg, Haimbfüerung. Uff was Örtter man im Hin-und Widerraisen zukommen, sampt einer Verzeichnus der Personen und Pferd, so zu Dessau erschinen, beschriben durch Georg Steglin. 1571 (Der ursprüngliche Titel lautete „Anhaltische Haimbfüerung“). HStA Stuttgart G 54 Bü 4.

7 Ebd., Bl. 13a $-16^{\mathrm{b}}$. 
einer Galerie umgebenen Lusthaus gab es neben anderen Räumlichkeiten einen schönen Sal uff die welsche Art. ${ }^{8}$

Das Schloss in Arnstadt war gar nach welscher oder französischer Manier mit schönen Erckern und Gengen im Schlo $\beta$ versehen. Es hatte einen viereckigen Hof, und an jeder Ecke befand sich wie in Kassel eine Wendeltreppe. Das Schloss war in- und auswendig mit schönen Malereien verziert und hatte herrliche Säle. Stegle glaubte nicht, dass es in Deutschland ein schöneres Grafenschloss gebe, und dasselbe sagte er auch von dem angrenzenden sehr großen Garten. Dieser wurde von einem Bach durchflossen, der sich teilte und wieder vereinigte, und auf der dadurch gebildeten Insel stand ein schönes Lusthaus. In dem Garten befand sich neben einem Vogelhaus, dem Gärtnerhaus und anderen Gebäuden auch der eigentliche, in gevierdte Compartementa unterteilte Lustgarten mit schönen Pflanzen und Hecken dazwischen. ${ }^{9}$

Sicher sammelte Stegle auf dieser Reise, die zwei Monate dauerte, wichtige Erfahrungen für seine Tätigkeit als Baumeister. Von Bedeutung ist auch, dass er mit den neueren, von Frankreich ausgehenden Strömungen in Berührung kam, was Ausdrücke wie „nach welscher oder französischer Manier“ belegen. Sein größtes Projekt wurde später das unter Wolfgang von Hohenlohe erbaute Schloss Weikersheim.

\section{Graf Wolfgang von Hohenlohe in Langenburg}

Graf Wolfgang II. von Hohenlohe wurde 1546 in der hohenlohischen Residenz Waldenburg geboren. Sein Vater war Graf Ludwig Casimir von Hohenlohe, seine Mutter, Gräfin Anna, eine geborene Gräfin von Solms-Laubach. Als Zwölfjähriger besuchte er zusammen mit seinem älteren Bruder Albrecht für zwei Jahre die Universität Tübingen, begab sich dann zu einem zweijährigen Studienaufenthalt nach Paris und war schließlich in Wien mehrere Jahre im kaiserlichen Dienst tätig. Von Wien aus nahm er auch an einem Feldzug gegen die Türken in Ungarn teil. Nach seiner Rückkehr in die Heimat nach Neuenstein vermählte er sich mit Magdalena, Gräfin von Nassau-Katzenelnbogen, einer Schwester Wilhelms von Oranien.

Als Wolfgangs Vater 1568 starb, übernahm für die nächste Zeit Gräfin Anna mit ihren beiden ältesten Söhnen Albrecht und Wolfgang die Regierung. 1573 wurde nach langen Beratungen eine Assignationsteilung vorgenommen. Albrecht bekam den Landesteil Weikersheim, Wolfgang den Teil Langenburg. 1574 bezog Wolfgang mit seiner Familie seine Residenz in Langenburg. Albrecht verunglückte im November 1575 bei einem Turnier tödlich, das in Stuttgart anlässlich der Vermählung von Herzog Ludwig mit Dorothea Ursula, Markgräfin von Ba-

8 Ebd., B1. $41^{\mathrm{a}}-42^{\mathrm{b}}$.

9 Ebd., Bl. $43^{\mathrm{a}}-45^{\mathrm{a}}$. 
den, veranstaltet wurde; sein Turniergegner war Joachim Ernst von Anhalt. ${ }^{10}$ Mit seiner Mutter einigte sich Wolfgang über die Aufteilung des Erbes und erhielt Schloss und Amt Weikersheim zur provisorischen Verwaltung.

Im April 1582 begannen die Vorbereitungen für eine definitive Landesteilung zwischen Wolfgang und seinen Brüdern Philipp und Friedrich, die im Juni 1586 im Saal der Weikersheimer Wasserburg vollzogen wurde. Durchs Los erhielt Wolfgang den Landesteil Weikersheim, Philipp Neuenstein und Friedrich Langenburg. Im März 1587 verlegte Wolfgang seine Residenz von Langenburg nach Weikersheim. ${ }^{11}$ Er starb 1610 in Weikersheim.

Am 13. Juni 1575 wandte sich Wolfgang an den in Ypern geborenen Baumeister Georg Robin (Robyn), der seit dem 28. Februar dieses Jahres im Dienst des Kurfürsten von Mainz stand. ${ }^{12}$ Wolfgang bat ihn, am 24. Juni nach Langenburg zu kommen, da er dort einen Neubau errichten wollte und Robin die hierfür notwendigen „Bausachen“ besichtigen sollte. Robin antwortete, dass er zu dem angegebenen Termin nicht kommen könne, da er in Mainz im Auftrag des Kurfürsten zwei größere Bauten begonnen habe - es handelte sich um die Kanzlei und die St. Gangolfkirche. Der Kurfürst habe aber erlaubt, dass er in etwa sechs Wochen kommen könne. Offenbar war Robin dann im Laufe des Sommers in Langenburg, vermaß die alte Anlage und entwarf eine neue Planung, obwohl dies nicht aktenmäßig belegt ist.

Wolfgang zog auch einen anderen Baumeister, Ulrich Unsinnig, zu Rate, der gerade Bauarbeiten auf der Burg Tierberg ausführte. Er kam zweimal nach Langenburg und machte Verbesserungsvorschläge zu dem Entwurf von Robin. Am 20. Januar 1576 schickte Wolfgang den ,ganzen Abriss“ an Robin. Die Ausarbeitung des Entwurfs sollte der Langenburger Schreinergeselle Thomas Fendrich übernehmen, der in Wolfgangs Dienst stand. Er schickte Fendrich zu Robin in die Lehre, der ihn in allem, was mit dem Bauwesen zusammenhängt, unterrichten sollte. Am 1. Februar 1576 schrieb Fendrich aus Mainz an Wolfgang, dass er von Robin sehr freundlich aufgenommen worden sei.

In Mainz hatte Fendrich von Robin auch den Auftrag, ein Modell (,,aufrechte Visierung“) aus Holz für den geplanten Neubau von Schloss Langenburg anzufertigen. Wolfgang bat Robin, dass Fendrich die Arbeit an dem Modell mit aller Sorgfalt ausführen solle. Das Modell war zu Ostern noch nicht fertig. Fendrich erklärte die Verzögerung in einem Brief an Wolfgang damit, dass der Kurfürst für die Kanzlei und die St. Gangolfkirche ein Modell aus Holz wünschte und

10 Adolf Fischer: Geschichte des Hauses Hohenlohe. 2. Teil, 1. Hälfte. Stuttgart 1868, S. 94-97. 11 Jost Weyer: Graf Wolfgang II. von Hohenlohe und die Alchemie. Alchemistische Studien in Schloß Weikersheim 1587-1610 (FWFr 39). Sigmaringen 1992, S. 23 f.

12 Max H. von Freeden: Zum Leben und Werk des Baumeisters Georg Robin. In: Zeitschrift für Kunstgeschichte 11 (1943-1944), S. 28-43. - Die meisten der von ihm im Hohenlohe-Zentralarchiv ausgewerteten Archivalien haben heute die Signaturen We 50 Bü D 6b (Tätigkeiten des kurfürstlich mainzischen Baumeisters Georg Robin in Langenburg) und We 50 Bü D 6c (Arbeiten des Schreiners Thomas Fendrich aus Mainz in Langenburg). 
dass Robin Fendrich mit dessen Anfertigung beauftragt hatte. Es dauerte dann aber doch noch einige Monate mit der Fertigstellung. Ende September wurde das große Schlossmodell mit dem Schiff nach Miltenberg transportiert und von dort mit dem Wagen über Land nach Langenburg, begleitet von Fendrich und Robins Stiefsohn.

Wolfgang, der sich in dieser Zeit bei Herzog Ludwig aufgehalten hatte, bedankte sich nach seiner Rückkehr nach Langenburg in einem Brief vom 11. Oktober bei Robin. ${ }^{13}$ Das Modell sei zu seiner vollen Zufriedenheit ausgefallen. Nach der Frage der angefallenen Kosten kommt er auf ein anderes Thema zu sprechen. Er wolle nicht verbergen, dass sich zwischen uns unnd unsern jungen Gebrüdern allerhanndt Verenderung zugetragen. Derowegen und weil wir noch nicht eigentlichs wissen, an welchem Ort wir unnsere bestendige Hoffhaltung anrichten werden, seindt wir nicht bedacht, innerhalb zweyen Jaren an unnßerm Bau anfahen zu lassen. Er benötige daher den Schreiner in dieser Zeit nicht, sondern er könne noch im kurfürstlichen Dienst bleiben.

Die zitierte Stelle spiegelt die Tatsache wider, dass nach dem Tod seines Bruders Albrecht am 16. November 1575 eine neue Situation eingetreten war. Wolfgang hatte Schloss und Amt Weikersheim zur provisorischen Verwaltung erhalten, und er konnte sich ausrechnen, dass es über kurz oder lang mit seinen Brüdern Philipp und Friedrich zu einer Erbeinigung kommen musste. Erst dann würde er wissen, welcher Ort seine endgültige Residenz sein werde. Folglich unterblieben in den nächsten Jahren alle größeren Bauarbeiten, und es wurde nur das ausgeführt, was unbedingt nötig war. Dazu gehörten einige Wirtschaftsgebäude und Ställe, eine Kinderstube, ein Glockenturm, der Archivraum, zwei Pulvertürme und ein etwas größerer Bau. ${ }^{14}$

\section{Graf Wolfgangs Pläne für den Bau von Schloss Weikersheim}

Am 3. Juli 1583 teilte Wolfgang seiner in Neuenstein lebenden Mutter Anna brieflich mit, dass er nicht nur in Langenburg, sondern auch in Weikersheim zu bauen gedenke. ${ }^{15}$ Dieser neue Gedanke ist zweifellos darauf zurückzuführen, dass 1582 die Vorbereitungen für eine definitive Landesteilung begonnen hatten, wobei Wolfgang noch nicht wissen konnte, ob er Weikersheim als endgültige Residenz erhalten würde. Der entsprechende Abschnitt in dem Brief lautet: Nachdeme die Notturfft erfordern thut, nicht allein alhie im Schloß Langenberg, sonder auch zue Weickhersheim zue bauen, habe ich den Sachen nachgedacht und befinde, daß nicht hinderslich, wan man solcher Beu zu Weickhers-

13 HZA We 50 Bü D 6 b.

14 Gerhard Taddey: Neue Forschungen zur Baugeschichte von Schloß Langenburg. In: WFr 63 (1979), S. 13-46.

15 HZA We 50 Bü D 12. 
heim durch einen Werckhmeister besichtigen und dieselbe in ein Visirung stellen und fassen liesse.

Er habe, so schreibt Wolfgang, an den Baumeister Georg Robin gedacht, der mit solchen Dingen gut vertraut sei. Er meine, dass man mit dem Rat der Baumeister und solchen Visierungen sich viele Unkosten und Mühe ersparen könne, und wollte den Rat seiner Mutter hierzu wissen. Ein Kanzlist vermerkte, dass der Brief nicht abgeschickt, sondern bei einem Besuch in Langenburg mündlich besprochen worden sei und seine Mutter damit einverstanden war.

Am 19. Juli wandte sich Wolfgang an den Kurfürsten von Mainz und bat um Beurlaubung von Robin. ${ }^{16}$ Er habe sich entschlossen, in seiner Grafschaft und der seiner Brüder in etlichen [...] Schlosser und Heusern allerhandt Beue ins Werckh zu richten. Ähnlich knapp fällt die Bezeichnung des Bauvorhabens in dem Brief aus, den Wolfgang noch am selben Tag an Robin schrieb. ${ }^{17}$ Er habe vor, etliche Beu in unser Graveschafft Heusern ins Werckh richten und ufbauen [...] zu lassen. Robin schrieb am 30. September, dass er erst in vierzehn Tagen nach Langenburg kommen könne, musste aber Anfang November mitteilen, dass ihm wegen eigener Arbeiten erst jetzt ein Kommen möglich sei. Da Wolfgang aber wegen Erbschaftsangelegenheiten nach Arnstadt reisen musste, wurde in diesem Jahr nichts mehr aus einem Treffen. ${ }^{18}$ Die Korrespondenz zwischen Wolfgang und Robin bricht dann für drei Jahre ab, so dass Robin auch später nicht nach Langenburg oder Weikersheim gekommen ist. Arnstadt ist übrigens derselbe Ort, dessen teilweise „,nach welscher oder französischer Manier“ gestaltetes Schloss Stegle in seiner „Anhaltischen Heimführung“ in so leuchtenden Farben beschrieben hatte.

Etwa um diese Zeit, spätestens aber mit der Landesteilung im Jahr 1586, muss Wolfgang konkretere Ideen über den Bau von Schloss Weikersheim entwickelt haben. Eine seiner beiden Grundideen ist die, dass der Grundriss des Schlosses die Form eines gleichseitigen Dreiecks haben sollte. Das Herzstück dieser Schlossanlage - und dies ist die zweite Grundidee - sollte ein großer Saal mit einer freitragenden Decke bilden. Ob auch die Ideen von Gängen oder Korridoren mit davon abgehenden Räumen und von einer großen zweiläufigen Steintreppe auf ihn zurückgehen, bleibt offen.

An dieser Stelle muss auf zwei grundlegende Veröffentlichungen von WaltherGerd Fleck über die Baugeschichte von Schloss Weikersheim hingewiesen werden. In seiner Dissertation, von der ein maschinenschriftliches Exemplar in der Universitätsbibliothek Tübingen aufbewahrt ist, untersucht er die Thematik an Hand des Quellenmaterials ausführlich und bringt bei strittigen Fragen die Beweise. ${ }^{19}$ In seiner Monographie über dieses Thema, durch zahlreiche Zeichnun-

16 Ebd. We 50 Bü D 6b.

17 Ebd.

18 Von Freeden (wie Anm. 12), S. 37.

19 Walther-Gerd Fleck: Das Schloß Weikersheim. Seine Baugeschichte und seine Stellung inner- 
gen illustriert, fasst er die Ergebnisse zusammen und verweist in den Fußnoten immer wieder auf den ausführlichen Text in der Dissertation. ${ }^{20}$ In beiden Veröffentlichungen widmet er sich auch der Schlossbaukunst jener Zeit, als Schloss Weikersheim entstand.

Wichtige Impulse für den Schlossbau im 16. Jahrhundert gingen von Italien und Frankreich aus. Entsprechend dem Streben der Renaissance nach Regelmäßigkeit experimentierten die Architekturtheoretiker in ihren Schriften außer den üblichen Rechtecken und Quadraten auch mit ungewöhnlichen geometrischen Grundrissen. Die wohl bedeutendste dieser Veröffentlichungen ist das Werk „D’Architecture“ von Jacques I. Androuet Du Cerceau (1559), das auch die Zeichnung eines Schlosses mit dreieckigem Grundriss enthält. ${ }^{21}$ In Frankreich gab es auch einige konkrete Beispiele für Schlösser mit dreieckigem Grundriss: Poitiers, Mehun-sur-Yèvre und Rambouillet. ${ }^{22}$

Der wichtigste Raum eines Renaissanceschlosses war der große Saal. Von Bedeutung war die Raumwirkung, d. h. das Verhältnis von Länge, Breite und Höhe. Viele der Säle gingen über nur ein Stockwerk und machten deshalb einen gedrückten Raumeindruck. Bei einigen Schlössern lag der große Saal dezentral, bei anderen nahm er einen ganzen Flügel des Schlosses ein, und bei einer dritten Gruppe lag der Saal im Hauptflügel, nahm diesen aber nicht ganz ein. ${ }^{23}$ Die Idee eines Saales in den Ausmaßen, wie er später geplant und realisiert wurde, mit einer freitragenden Decke war etwas Außergewöhnliches.

Wolfgang war, wie viele seiner fürstlichen Zeitgenossen, ein baufreudiger Herrscher und wollte hinter ihnen nicht zurückstehen. Ein Beleg hierfür ist sein Bücherverzeichnis, das er in Langenburg anfertigen ließ, wahrscheinlich im Jahr 1586, als der Umzug nach Weikersheim bevorstand. ${ }^{24}$ Es enthält eine Rubrik mit etwa 25 Bänden über Architektur, Perspektive, Geometrie, Sonnenuhren, Gebäudemessung, Feldmessung, Feld- und Gartenbau. Unter den Titeln zur Architektur findet man auch das Werk „De Architectura Jacobi Andronetij [sic] du Cerceau opus“, was bisher von keinem Autor beachtet wurde. ${ }^{25}$ Es ist die lateinische Version des „Livre D’Architecture“ von Du Cerceau, die im selben Jahr wie

halb der Schloßbaukunst des 16. und des frühen 17. Jahrhunderts. Diss. phil. (maschinenschriftlich). Tübingen 1952.

20 Walther-Gerd Fleck: Schloß Weikersheim und die hohenlohischen Schlösser der Renaissance (Tübinger Forschungen zur Kunstgeschichte 8). Tübingen 1954.

21 Jacques Androuet Du Cerceau: Livre D'Architecture De Jaqves Androvet du Cerceau. Contenant les plans \& dessaings de cinquante bastimens tous differens ... Paris 1559. - Second Livre d'Architecture. 1561. - [Troisieme] Livre d'Architecture. 1582.

22 Fleck (wie Anm. 20), S. 23 f.

23 Ebd., S. $25 \mathrm{f}$.

24 Verzeichnis der Bücher in Wolfgangs von Hohenlohe Bibliothek in Langenburg, undatiert, wahrscheinlich 1586. HZA Gemeinschaftliches Archiv 75, Bd. J 1.

25 Bücherverzeichnis (wie Anm. 24), S. 53. 
die französische Ausgabe erschien. ${ }^{26}$ Auf Grund dieses Befundes liegt die Folgerung nahe, dass Wolfgang durch das Werk von Du Cerceau zu der Idee einer Dreiecksanlage angeregt wurde. Schloss Rambouillet hatte er während seines Studienaufenthalts in Paris mit Sicherheit kennengelernt. Auch sonst hatte Wolfgang als junger Mann und später auf seinen Reisen, wie man annehmen darf, manche Schlösser gesehen und dabei Anregungen für den eigenen Schlossbau gesammelt.

Wolfgang von Hohenlohe war einer der bedeutendsten Herrscher aus dem Geschlecht der Hohenlohe und seinen fürstlichen Kollegen mit einem größeren Territorium durchaus ebenbürtig. Auch das später im Rittersaal verwirklichte Bildprogramm mit dem Eingangsportal, dem Prunkkamin und der Ahnentafel ${ }^{27}$ sowie den Deckenbildern ${ }^{28}$ lässt erkennen, dass dahinter eine markante Persönlichkeit stand. Unter diesen Voraussetzungen ist es nicht denknotwendig, dass Wolfgang durch einen Baumeister auf die Idee einer Dreiecksanlage gebracht wurde, zumal die Anregung durch das Werk von Du Cerceau wahrscheinlich gemacht wurde.

Am 3. Juni 1586 fand im Saal der alten Weikersheimer Burg der offizielle Akt der definitiven Landesteilung statt. Die Namen der drei Teilgrafschaften wurden auf Zettel geschrieben, die Zettel zusammengefaltet, verschlossen und in einen Hut gelegt. Ein vierzehnjähriger Junge aus Weikersheim zog für jeden der drei Brüder die Lose, und so kam Weikersheim an Wolfgang, Neuenstein an Philipp und Langenburg an Friedrich.

Auf diese Entscheidung muss Wolfgang schon ungeduldig gewartet haben endlich konnte er seine Bauvorhaben verwirklichen. Am 16. Juli schrieb er an Robin, dass ihm vor kurzem durch Erbteilung mit seinen Brüdern unter anderem das Hauß und Ambt Weickhersheim zugefallen sei und es nun nötig sei, dort und an anderen Stellen zu bauen. ${ }^{29}$ Er wolle solchen Bau gern ordenlich abgerissen und in ein Visirung gebracht haben. Am 28. Juli teilte er dem Kurfürsten von Mainz mit, dass er vorhabe, einen nothwendigen Bau an meinem Schlo $\beta$ Weickhersheim demnechst kunffigen Sommer vorzunhemen, und bat den Kurfürsten, Robin wegen eines Gutachtens für „etliche Tage“ zu beurlauben. ${ }^{30}$ Eine Antwort kam weder von Robin noch vom Kurfürsten, da Robin längerfristig mit dem Schlossbau in Höchst beschäftigt war. ${ }^{31}$

26 Jacques Androuet Du Cerceau: De Architectvra Jacobi Androvetii Opus. Quo descriptae sunt aedificiorum quinquaginta planè dissimilium ichnographiae ... Paris 1559.

27 Jürgen Kniep: „Gott gibt Glück“. Graf Wolfgang II. von Hohenlohe und die politisch-religiöse Symbolik im Rittersaal von Schloss Weikersheim. In: WFr 89 (2005), S. 39-74.

28 Jakob Käpplinger: Die Jagd als Spiegel der Gesellschaft. Bemerkungen zum Raumprogramm des Rittersaals in Schloss Weikersheim. In: WFr 95 (2011), S. 73-93.

29 HZA We 50 Bü D 6 b.

30 Ebd.

31 Von Freeden (wie Anm. 12), S. 37. 
Wolfgang wandte sich daraufhin am 22. November an Stegle, mit dem er wegen anderer Bauvorhaben schon seit 1583 in Kontakt gestanden hatte, und bat ihn, zu einem Gespräch von unser vorhabenden Baues Sachen am 8. Januar nach Langenburg zu kommen. ${ }^{32}$ Eine Beurlaubung von Stegle durch seinen Dienstherren versprach Erfolg, da Wolfgang und Ludwig von Württemberg befreundet waren, sich wiederholt in Stuttgart trafen und auf der Schwäbischen Alb oder im Schönbuch gemeinsam auf die Hirschjagd gingen. Die guten Beziehungen zwischen den Häusern Württemberg und Hohenlohe demonstriert auch das zentrale Deckenbild im Rittersaal. Dort ist die Jagd auf den weißen Hirsch dargestellt, und im Hintergrund sieht man die Burg Württemberg in der Nähe von Stuttgart, den Stammsitz der Herzöge von Württemberg. ${ }^{33}$

Über die Korrespondenz von Wolfgang mit Stegle und Ludwig betreffs Schloss Weikersheim hat Fleck ausführlich berichtet. ${ }^{34}$ Die 25 Briefe handeln von Bitten um das Kommen, Vertröstungen, später Anmahnungen Ludwigs und Bitten Wolfgangs um Verlängerung seines Aufenthalts in Weikersheim. Hier sollen nur die Briefe näher untersucht werden, in denen etwas über Wolfgangs Bauvorhaben und speziell über die Bauzeichnungen und das Modell ausgesagt wird. Am 14. März 1587 verlegte Wolfgang seine Residenz von Langenburg nach Weikersheim, so dass alle weitere Korrespondenz von dorther kam oder dorthin ging. Am 26. Mai beauftragte er den Zimmermeister Lienhard Niebel als Baumeister mit dem Umbau des Beamtenbaus, damit Wolfgang mit seiner Familie einigermaßen standesgemäß untergebracht war und die Verwaltungs- und Regierungsgeschäfte ausgeführt werden konnten. Dies war bereits Anfang 1588 der Fall. ${ }^{35}$

Am 12. Januar 1588 schrieb Wolfgang an Stegle, dass er vorhabe, einen Hauptbau ze thun, und bat ihn, sich in Stuttgart nach einem guten Zimmermann für das Hängewerk umzusehen. ${ }^{36}$ Der „Hauptbau“ war der spätere Saalbau, und das Hängewerk war für die freitragende Decke des Saales bestimmt. Stegle empfahl Jacob Saltzmann als Zimmermann und schickte dessen Risse, da dieser nicht selbst kommen konnte. Am 30. Januar erinnerte Wolfgang Stegle daran, dass er mit Einverständnis des Herzogs in 14 Tagen nach Weikersheim kommen solle, da wir dan jetzo euer zue Raissung einer Visierung gantz nothwendig weren. ${ }^{37}$ Stegle antwortete am 12. Februar, dass er wegen eines nötigen Baus auf dem Hohenasperg keinen Urlaub erhalten habe. ${ }^{38}$ In diesem Brief schickte er auch die erwähnten Risse von Saltzmann. Er unterschrieb - wie auch in den anderen Brie-

32 HZA We 50 Bü D 6e.

33 Walther-Gerd Fleck: Eine Darstellung der Burg Württemberg in Schloß Weikersheim. In: ZWLG 49 (1990), S. 437-440.

34 Fleck (wie Anm. 19), S. 46 f.

35 Ebd., S. $44 \mathrm{f}$.

36 HZA (wie Anm. 32).

37 Ebd.

38 Ebd. 


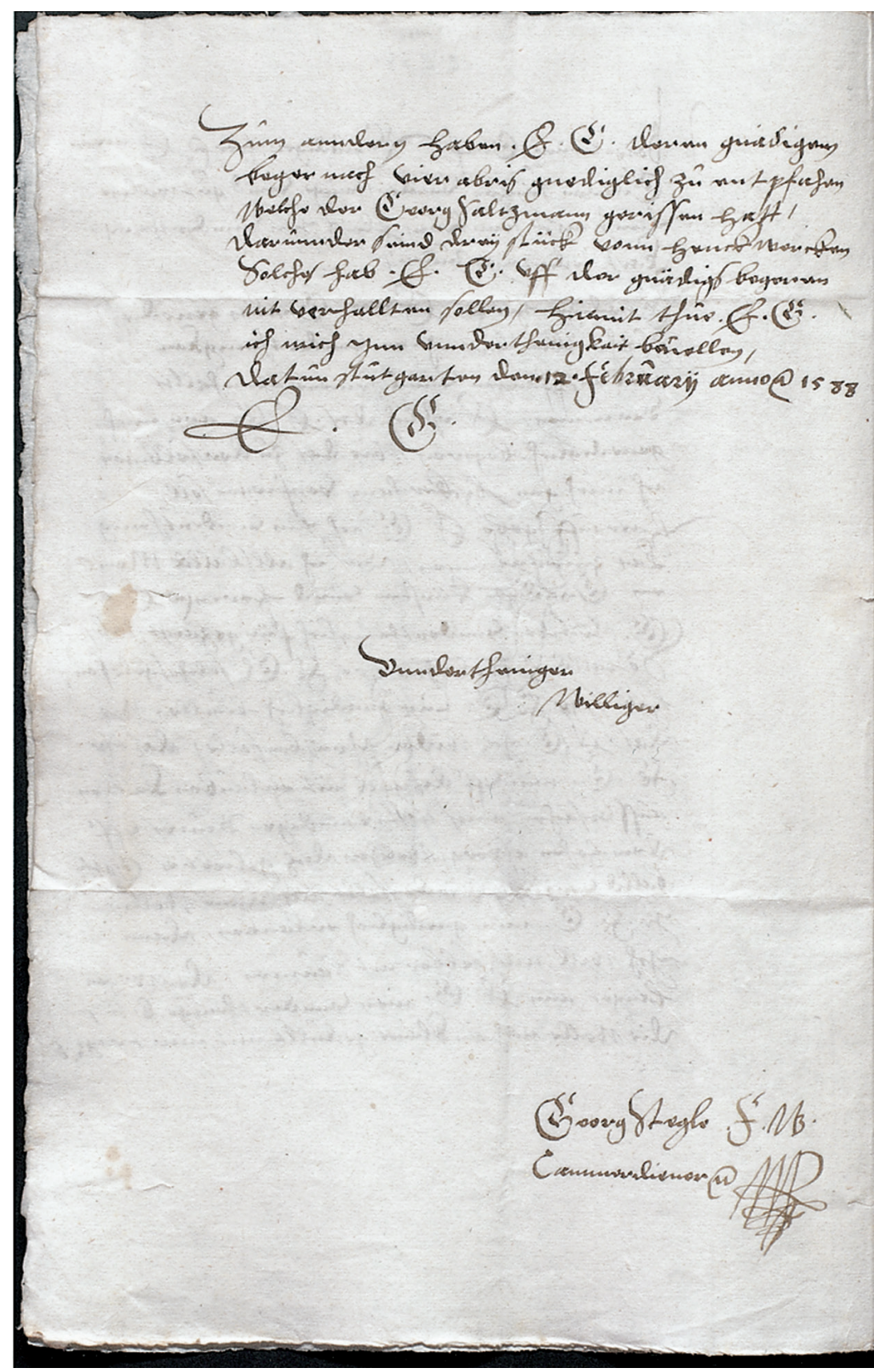

Abb. 1 Seite aus einem Brief von Georg Stegle an Wolfgang von Hohenlohe mit Stegles Unterschrift (Hohenlohe-Zentralarchiv Neuenstein) 
fen - mit „Georg Stegle, Fürstlich Württembergischer Cammerdiener“ - Kammerdiener hier kein Lakai, sondern ein Ehrentitel. ${ }^{39}$

\section{Stegle zur Anfertigung von Bauzeichnungen und einem Modell in Weikersheim}

Am 3. August 1588 war es endlich so weit: An diesem Tag machte sich Stegle von Stuttgart auf den Weg nach Weikersheim, versehen mit der Bitte von Ludwig, Stegle nicht so lange aufzuhalten. ${ }^{40}$ Sein Aufenthalt in Weikersheim betrug dann, was keiner der drei voraussehen konnte, sieben Monate. In dieser Zeit fertigte Stegle die Bauzeichnungen und das Modell des Schlosses an. Da weder die Pläne noch das Modell heute noch vorhanden sind und es über die Gespräche zwischen Wolfgang und Stegle über den Bau selbstverständlich keine Aufzeichnungen gibt, müssen die Ergebnisse indirekt aus anderen Quellen abgeleitet werden.

Es handelte sich um einen völligen Neubau, in den von der alten Burg nur der Bergfried einbezogen werden sollte. Der Grundriss, der sich rekonstruieren ließ, war ein gleichseitiges Dreieck mit abgeplatteten Ecken. ${ }^{41}$ Dominierend war der große Saal in der Mitte der einen Dreiecksseite. Der einzige Schmuck des Schlosses nach außen hin waren einige Ziergiebel. In den beiden anderen Seiten des Dreiecks befanden sich Gänge, von denen die Räume abgingen, mit Fenstern zum Schlossinnenhof hin.

Dass die Ideen des dreieckigen Grundrisses und des großen Saales mit der freitragenden Decke von Wolfgang ausgingen, kann als gesichert gelten. Sonst aber ist nicht mehr zu ermitteln, welche Anregungen von Wolfgang und welche von Stegle kamen. Fleischhauer schreibt: Stegles Fähigkeiten und damit auch seine Einwirkung auf den bedeutenden Weikersheimer Bau sollen nicht unterschätzt werden. ${ }^{42}$ Anregungen und Vorbilder hatte er durch die Bauten seiner Kollegen von der Stuttgarter Bauschule. Die Giebel haben ihr Vorbild in den von Blasius Berwart entworfenen Giebeln des Ansbacher Schlosses. Das Hängewerk über dem Saal wurde in der Bauphase von Elias Gunzenhäuser entworfen, der einige Jahre vorher das Stuttgarter Neue Lusthaus mit einem großen Hängewerk versehen hatte. Vorbild für die zweiläufige Steintreppe war zweifellos die Reittreppe im Stuttgarter Alten Schloss. Dies war eine Neuerung, denn auch in der Renaissance noch war es üblich, die Stockwerke durch Wendeltreppen zu verbinden. ${ }^{43}$ Eine andere wichtige Neuerung, die Gänge mit davon abgehenden Räumen, dürfte Stegle bei der „Anhaltischen Heimführung“ - wenn die Interpretation mit

39 Siehe Abb. 1.

40 HZA (wie Anm. 32).

41 Siehe Abb. 2.

42 Fleischhauer (wie Anm. 2), S. 81.

43 Fleck (wie Anm. 20), S. 12 f., 18-41. 


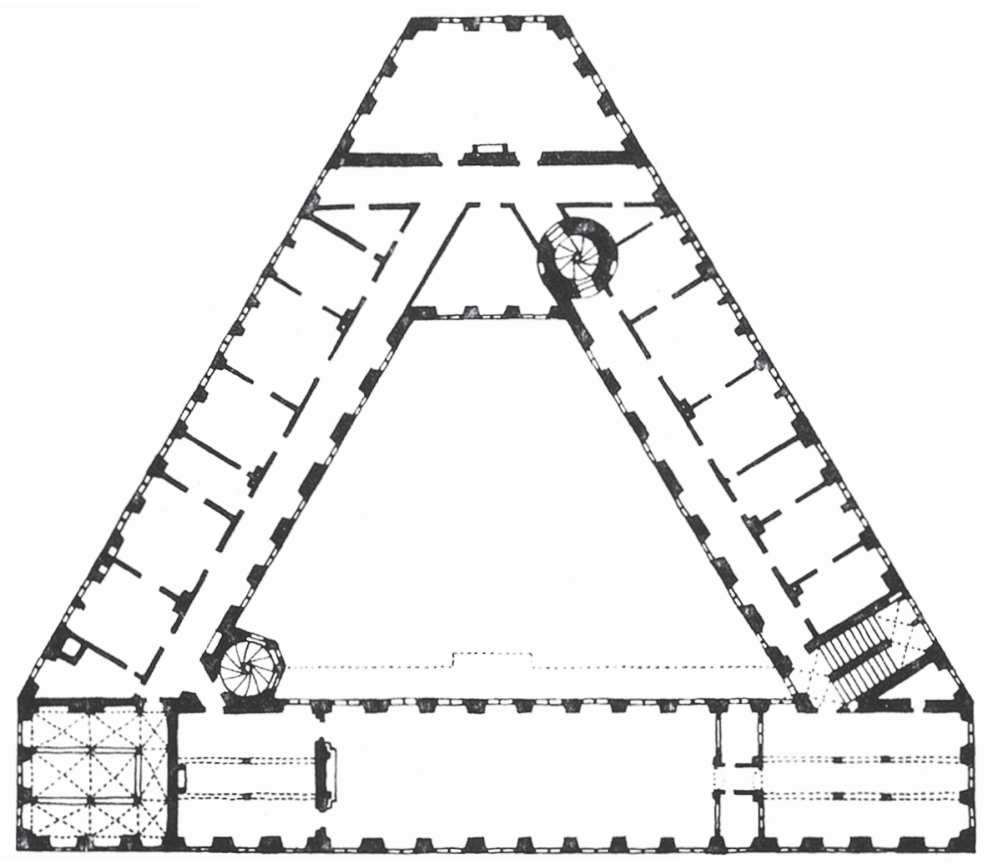

Abb. 2 Schloss Weikersheim, Grundriss des 1. Stockwerks nach dem Plan von Georg Stegle (Zeichnung: Walther-Gerd Fleck, Stuttgart)

den „Gängen“ zutrifft - in Schloss Arnstadt kennengelernt haben, das auch Wolfgang bekannt war.

Was das Aussehen des Schlossmodells betrifft, so lassen sich aus der Korrespondenz von Wolfgang mit Robin und Fendrich über das Modell von Schloss Langenburg ein paar Informationen entnehmen. ${ }^{44}$ Es musste ein besonderes Holz sein, das zurechtgesägt und mit dem Hobel bearbeitet wurde. Am 8. Februar 1576 schrieb Wolfgang an Fendrich nach Mainz: Auch wollest die Thor unnd Fenster, wie die gehauen werden sollen, alles schnitzeln und zum fleißigsten machen. ${ }^{45}$ Das Modell muss also so groß gewesen sein, dass er eine feine Herausarbeitung der Türen und Fenster verlangen konnte. In einem Schreiben vom 10. März wandte er sich an Robin. ${ }^{46}$ Er solle darauf achten, dass bei der von Fendrich ausgeführten ,aufgerichten Visierung“ auch die Stein- unnd Holtzwerckh allerdings mit dem Mahlen unnd Anstreichenn, wie sie sein sollen, zugerichtet werde. Es wurden also die Stein- und Holzteile des vorgesehenen 
Neubaus in den entsprechenden Farben koloriert. Anschließend wurden die Einzelteile des Modells aufgerichtet und verleimt.

Einige sehr spärliche Informationen über das Modell enthält die Korrespondenz, die Wolfgang mit Ludwig während der Anwesenheit von Stegle in Weikersheim führte. Am 25. September 1588 - also etwa anderthalb Monate nach Stegles Ankunft - teilte Wolfgang mit, dass er nicht geglaubt habe, daß die Sachen und Visirung so lange gedauert hätten. ${ }^{47}$ Demnach aber er mir das vorhabende Werckh ufrecht in Holtz zue machen in der Arbeit, was noch etwa drei Wochen dauern könne, bitte er um weitere Beurlaubung. Am 7. März 1589 konnte Wolfgang endlich mitteilen, dass Stegle die Arbeit zue Verfertigung meiner Visirung beendet habe, und dankte Ludwig für dessen Beurlaubung über eine so lange Zeitspanne. ${ }^{48}$

Die Bedeutung der Rolle von Stegle bei der Bauplanung von Schloss Weikersheim wurde von Freeden in Frage gestellt. ${ }^{49}$ Er konnte sich offenbar nicht vorstellen, dass die Baupläne für Schloss Weikersheim mit ihren teils außergewöhnlichen Ideen von Stegle stammten, sondern betrachtete Robin als den Urheber, der zweifellos der bedeutendere Baumeister war. Seine Argumente hierfür sind ausgesprochen schwach begründet. Auf Grund der Tatsache, dass sich Robin später in einem Brief über die Qualifikation von Meister Gilg, den Vorgänger von Niebel als Baumeister, negativ äußerte, folgerte er, dass Robin doch noch nach Weikersheim gekommen sei und die Entwürfe für den Neubau skizziert habe. Dabei war Robin wegen des Schlossbaus in Höchst noch 1587 nicht abkömmlich, und in der Korrespondenz Wolfgangs mit Robin fehlt jeder Hinweis auf einen solchen Aufenthalt. Freeden aber schreibt, dass die Bauarbeiten 1595 nach dem Plan Robins und dem Modell Stegles begannen.

Diese Interpretation übernahm auch Fleischhauer in seinem Werk über die Renaissance im Herzogtum Württemberg. ${ }^{50}$ Er schrieb: Stegles Pläne und Modell beruhten sicherlich im wesentlichen auf den Ideen von Robin. Max Hermann von Freeden (1913-2001), Direktor des Mainfränkischen Museums in Würzburg, war ein angesehener Kunsthistoriker. Es gab keinen Grund, seine Aussagen an den Quellen noch einmal nachzuprüfen. Verhängnisvoll wirkte sich aus, dass Freeden auch die ersten Auflagen des Schlossführers Weikersheim verfasste. So liest man beispielsweise in der 4. Auflage von 1954 die folgenden Sätze: „Der kurmainzische Baumeister Georg Robin [ ...]. entwarf den Plan für ein großes Renaissanceschloß. [...] Hofschreiner Stegle aus Stuttgart fertigte danach an Ort und Stelle das Modell in Holz und Gips". ${ }^{51}$ Er degradierte also Stegle zum Hofschreiner, ohne einen Beleg dafür zu haben, und machte ihn damit zum rein ausführenden Organ, der nichts mit der Planung zu tun hatte. Auch

47 Ebd., Bü D 6e.

48 Ebd.

49 Von Freeden (wie Anm. 12), S. $38 \mathrm{ff}$.

50 Fleischhauer (wie Anm. 2), S. 81.

51 Max H. v. Freeden: Schloss Weikersheim. München und Berlin ${ }^{4} 1954$, S. 2. 
als später andere Autoren den Schlossführer verfassten, übernahmen sie Freedens Interpretation. So schreibt Merten in der 4. Neuauflage von 1984: ,[Robin] legte auch wirklich einen höchst originellen Entwurf vor: auf dem Grundriß eines gleichseitigen Dreiecks sollte das neue Schloß [...] entstehen“. Und einige Zeilen weiter: „[...] der württembergische Hofschreiner Stegle, der das Modell hergestellt hatte [...]“. ${ }^{52}$ Das gedruckte Werk von Fleck über Schloss Weikersheim wird zwar in der Bibliographie aller Auflagen des Schlossführers aufgeführt, aber seine Interpretation wird nicht erwähnt, geschweige denn akzeptiert. Diese Situation hat sich bis auf den heutigen Tag nicht geändert.

In gewisser Beziehung hatte Freeden ja mit seinen Zweifeln Recht, ob die Idee des dreieckigen Grundrisses von Stegle stammte, aber er zog daraus die falschen Schlüsse. Fleck führte die Baupläne für Schloss Weikersheim auf Stegle zurück und belegte dies, soweit es quellenmäßig möglich war. Dennoch hatte auch Fleck seine Bedenken. In der Schlusszusammenfassung seiner Monographie sagt er, dass Stegle gewiss ein befähigter Meister war, der neben den Großen seiner Zeit und Umgebung ebenbürtig arbeitete. Er fährt dann fort: Trotzdem scheint damit die Entstehung eines Bauprojektes von solch einmaliger und zukunftweisender Gestaltung noch nicht hinreichend erklärt. Dies ist nur möglich durch die berechtigte Annahme, der Bauherr Graf Wolfgang II. von Hohenlohe, Herr zu Langenburg, habe selbst maßgebenden Anteil an der Planung gehabt und wohl hauptsächlich die neuen Grundideen beigesteuert. ${ }^{53}$ Diese Annahme von Fleck wurde nun durch den Nachweis von Du Cerceaus Werk über die Architektur in Wolfgangs Bibliothek bestätigt.

\section{Der Bau von Schloss Weikersheim}

Die Bauarbeiten für Schloss Weikersheim begannen erst im Frühjahr 1595. Vorher - von 1587 bis 1593 - wurden Baumaterialien für den Neubau geliefert und gestapelt: Holz, Bruchsteine, Ziegelsteine und Dachziegel. Für die Bauphase fehlen wichtige Akten, doch lässt sich der Ablauf aus den noch vorhandenen Archivalien und dem heutigen Baubestand rekonstruieren. Fleck hat dies alles in seiner Dissertation ausführlich beschrieben und belegt ${ }^{54}$ und in seiner Monographie stark verkürzt dargestellt. ${ }^{55}$

Hier soll zuerst die Korrespondenz Wolfgangs mit den Handwerkern und Baumeistern erörtert werden, dann der Verlauf der Bauarbeiten, obwohl beides zeitlich parallel liegt. Die Korrespondenz betrifft in erster Linie die Suche nach einem fähigen Baumeister für die Beaufsichtigung und Leitung der Bauarbeiten.

52 Klaus Merten: Schloß Weikersheim. München und Berlin ${ }^{4} 1984$. S. 4.

53 Fleck (wie Anm. 20), S. 41.

54 Fleck (wie Anm. 19), S. 48-63.

55 Fleck (wie Anm. 20), S. 10 ff. 
Das Fazit war, dass Wolfgang keinen Erfolg damit hatte, für den Neubau einen ständigen Baumeister zu gewinnen. Stattdessen standen ihm als führende Kräfte der Maurermeister Jacob Kauffmann aus Herzberg in Thüringen und der Steinmetzmeister Servatius Körber aus Bonn zur Verfügung. Am 1. Juni 1595 machte Wolfgang in einem Vertrag Kauffmann zum Aufseher über die am Bau tätigen Maurer und Taglöhner. ${ }^{56}$ Er war dann fast vierzig Jahre als Bauaufseher und Baumeister im hohenlohischen Dienst. ${ }^{57}$

Aus der Korrespondenz sollen nur diejenigen Schriftstücke ausgewählt werden, die Aussagen über die Baupläne und den Stand der Bauarbeiten gestatten. Zwei Schreiben mit wichtigen Informationen wurden am 18. Juni 1595 verfasst. Bei dem einen Dokument handelt es sich um einen Brief, den Wolfgang an den im württembergischen Dienst stehenden Zimmermann und Baumeister Elias Gunzenhäuser nach Stuttgart schrieb. ${ }^{58}$ Er bat ihn um die Empfehlung eines guten Meisters für das Hängewerk über dem großen Saal. Wolfgang schreibt: [...] das wir alhier an unserm Schloß einen großen Haubtbau zu dreyen Seitten, jede besonder ungevehr 250 Schuch lang, zu füern vorhabens, darein ein Saal, 23 Schuch hoch, über 100 Schuch lang und 40 Schuch breit, alles ohne Seulen, und auf solchen Saal drey underschiedliche Kornschüttungen kommen sollen. Der Passus zu dreyen Seitten, jede besonder ungevehr 250 Schuch lang ist in dem Briefentwurf, um den es sich hier handelt, gestrichen.

Dies war der Zeitpunkt, an dem Stegles Entwurf eines idealen Dreiecks mit der Realität konfrontiert wurde. Vielleicht aus praktischen Erwägungen heraus wurde sein Entwurf nur teilweise verwirklicht. ${ }^{59}$ Die Information über die Dreiecksanlage mit der Maßangabe - übrigens bisher die einzige quellenmäßig belegte Angabe Wolfgangs hierzu - wurde gestrichen, weil sie obsolet geworden war. Stattdessen gab er für den großen Saal schon die neuen Maße an. Fleck berechnete daraus, dass der Saal mehr als $30 \mathrm{~m}$ lang, $12 \mathrm{~m}$ breit und $6,9 \mathrm{~m}$ hoch war, wobei er $1 \mathrm{Schuh}=30 \mathrm{~cm}$ setzte ${ }^{60}$. Für die tatsächlichen Dimensionen des Saales ermittelte er durch Messung, dass er 36,4 $\mathrm{m}$ lang, 11,7 $\mathrm{m}$ breit und 8,25 $\mathrm{m}$ hoch ist. ${ }^{61}$ Vergleicht man den heutigen Grundriss mit dem von Stegle, dann hätte die Länge des ursprünglichen Saals bei identischer Breite 41,8 $\mathrm{m}$ betragen. Der heutige Saal ist also um 41,8 $\mathrm{m}-36,4 \mathrm{~m}=5,4 \mathrm{~m}$ in seiner Länge kürzer als der von Stegle geplante.

In seinem Brief hatte Wolfgang geschrieben, dass er bereit sei, einen solchen Meister, der auch etwas von dem Hängewerk verstehe, als Baumeister fest anzustellen. Gunzenhäuser war selbst daran interessiert, aber ein Vertrag kam wegen zu hoher finanzieller Forderungen nicht zustande. Er war in der zweiten Julihälf-

56 HZA We 50 Bü D 61.

57 Taddey (wie Anm. 14), S. 26, 29 f.

58 HZA We 50 Bü D $6 \mathrm{f}$.

59 Siehe Abb. 3 und 4.

60 Fleck (wie Anm. 20), S. 11.

61 Fleck (wie Anm. 19), S. 20. 

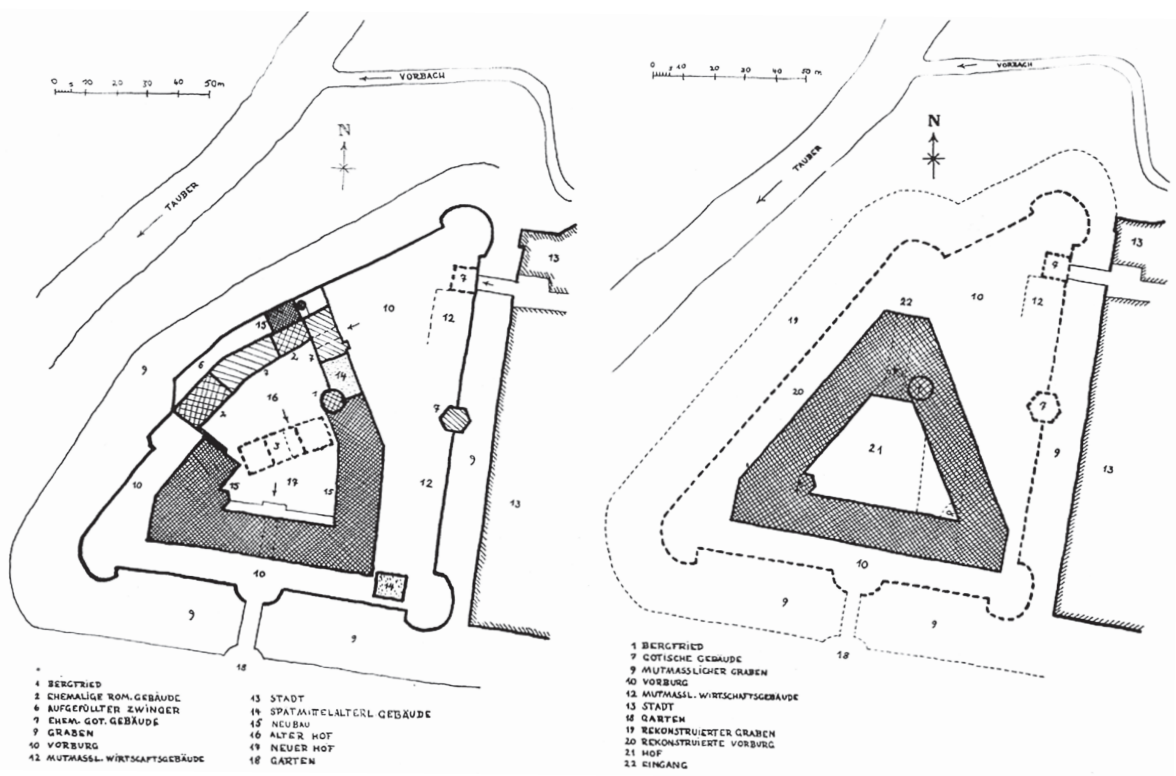

Abb. 3 und 4 Schloss Weikersheim, Bauzustand um 1610. -

Schloss Weikersheim nach dem Plan von Georg Stegle

(Zeichnung: Walther-Gerd Fleck, Stuttgart)

te für ein paar Tage in Weikersheim. In dieser Zeit wird er die Bauzeichnungen für das Hängewerk angefertigt haben, auch wenn dies nirgends dokumentiert ist. In den Bauphasen, als das Dach über den verschiedenen Gebäudeteilen aufgeschlagen wurde, war Gunzenhäuser als Baumeister von Herzog Ludwig nicht abkömmlich, so dass Wolfgangs Zimmerleute die Arbeiten allein ausführten. ${ }^{62}$ Das zweite Dokument vom 18. Juni betrifft ein Schreiben von Wolfgang an Wolfgang Beringer, der als Baumeister im Dienst des Fürstbischofs von Würzburg stand..$^{63}$ Aus diesem Brief soll ein längerer Abschnitt zitiert werden, da er ein lebhaftes Bild von Wolfgangs Situation in dieser Bauphase vermittelt. Er schreibt: [...] daß wir unsers bewußten Baus nachgedacht und nochmals gern ein gantze volkommene Visierung über den gantzen Bau, wie er allerdings werden solle, gehaben woltten, uff daß wir uns jedesmals darinnen nach gnugsamer Notturff ersehen und allenthalben unser Meinung erwegen mögen. Sintemal wir solches Zuthun nit alle Zeit Gelegenheit haben, noch erst uff das Uffmessen wartten können und wir allerley Gedancken haben, wie wir es gern an ainem und dem andern Ort machen woltten. Wan wir dan die Visirung nit gantz haben, sonder jedesmals nur das Stück, wie es vorgenommen wird, als 
anitzo geschihet, so verliern wir inn Mangel dessen und von wegen ander Geschefften unsere Gedancken, darumb wir hernacher gern viel geben woltten, daß wir es in der Visirung gemerckt hetten.

Da die Bauarbeiten um diese Zeit im vollen Gang waren und es erhebliche Abweichungen von Stegles Dreiecksplan gab, mussten immer wieder neue Bauzeichnungen angefertigt werden. Bei den vielen einzelnen Visierungen drohte der große Zusammenhang verloren zu gehen. Daher bat Wolfgang um die Anfertigung einer Visierung über den gantzen Bau, der den neuen Gegebenheiten entsprach. Der Text zeigt auch Wolfgangs Engagement bei der Planung. Er beließ es nicht nur bei der grundsätzlichen Abweichung von Stegles Plan, sondern kümmerte sich um jedes Detail. In der augenblicklichen Situation ohne einen Gesamtplan müsse er seine Gedanken immer wieder darauf konzentrieren, welche Ideen er im Einzelfall für den Neubau habe.

Ostern (20. April) 1595 hatte Wolfgang mit Beringer in Weikersheim einen Vertrag aufgesetzt, in dem er zum Baumeister für den alhier angefangenen neuen Bau bestallt wurde. ${ }^{64} \mathrm{Da}$ er als Baumeister bei dem Fürstbischof von Würzburg, Julius Echter von Mespelbrunn, fest angestellt war, brauchte er mit dessen Einwilligung nur dann nach Weikersheim zu kommen, wenn es notwendig war. Nach mehreren Bittbriefen kam Beringer endlich im August nach Weikersheim und blieb dort maximal vier Wochen. Mit Sicherheit fertigte er in dieser Zeit die dringend benötigten Zeichnungen des gesamten Neubaus an, die dem heutigen Bauzustand in den meisten Teilen ähnlich gewesen sein dürften. Im September wurde die Bestallung auf Beringers Wunsch aufgehoben. ${ }^{65}$

Die Bauarbeiten an dem im Frühjahr 1595 begonnenen Schlossbau schritten rasch voran. Zur besseren Veranschaulichung des im folgenden Beschriebenen sei auf den Grundriss hingewiesen, der den Bauzustand im Jahr 1610, dem Todesjahr Wolfgangs, zeigt. ${ }^{66}$ Links oben befindet sich der Beamtenbau, rechtwinklig daran anschließend der Prinzessinnenbau. Dann folgt vom ehemaligen Bergfried an mit einem Knick der Langenburger Bau, der mit der Reittreppe endet. Die Basis des Grundrisses bildet der Saalbau mit dem Rittersaal und der Schlosskapelle. Links schließt sich im Winkel der Küchenbau an, in der Ecke der Treppenturm mit Wendeltreppe. ${ }^{67}$ Stegles Plan mit einem dreieckigen Grundriss ist noch im oberen Teil des Langenburger Baus, im Saalbau und Küchenbau zu erkennen.

Der erste Bauabschnitt betraf den Langenburger Bau. Er wurde, wie gesagt, im Frühjahr 1595 begonnen und war im Juni 1596 im Rohbau fertig und unter Dach gebracht. Im Februar 1597 wurde mit dem Innenausbau begonnen, der wohl noch im selben Jahr beendet war. Der zweite Bauabschnitt umfasste die Reittrep-

64 Ebd.

65 Fleck (wie Anm. 19), S. 49 ff.

66 Siehe Abb. 5.

67 Die Bezeichnungen für diese Teile des Schlosses stammen alle aus späterer Zeit. 


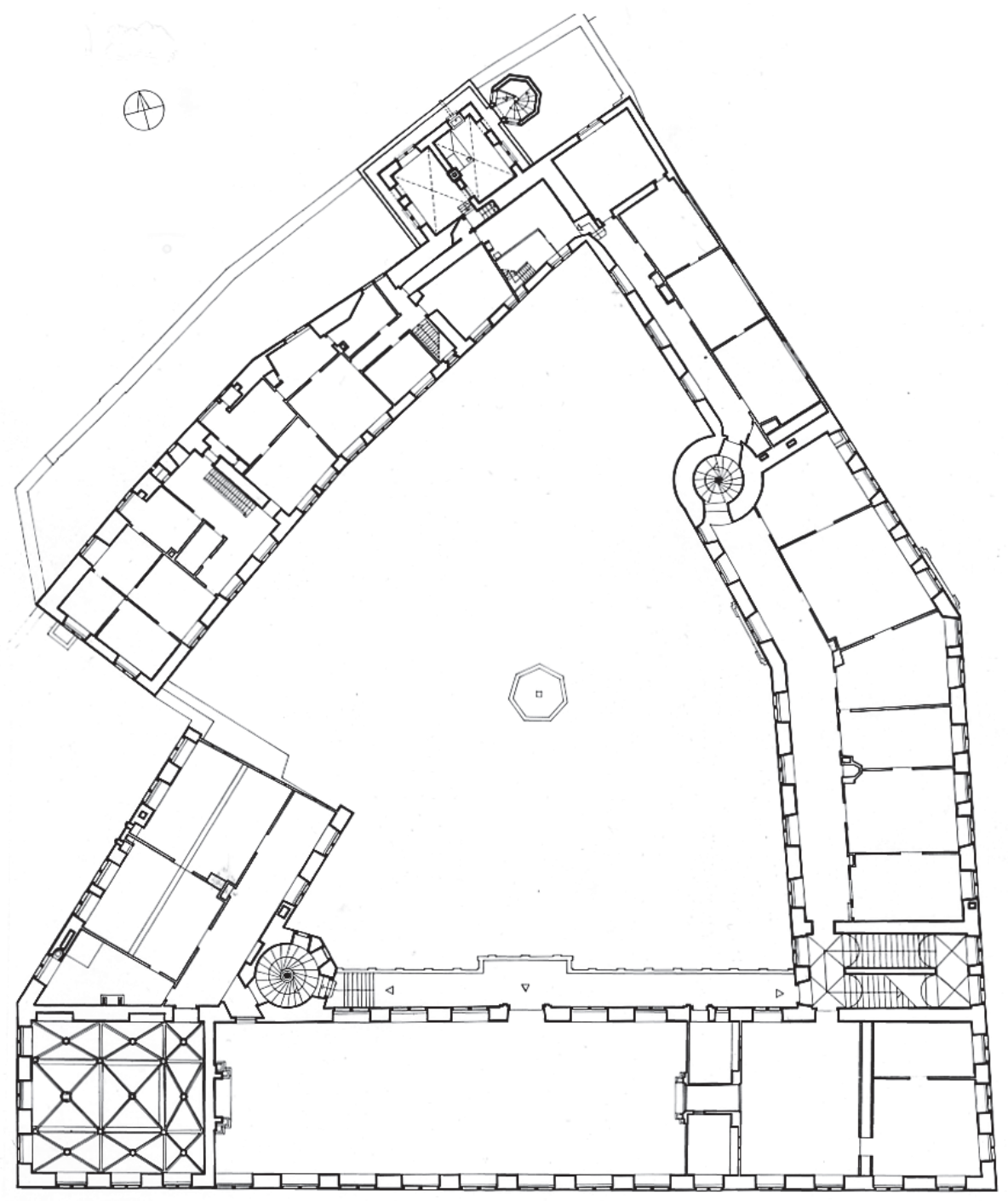

Abb. 5 Schloss Weikersheim, Grundriss des 1. Stockwerks, Bauzustand um 1610 (Zeichnung: Walther-Gerd Fleck, Stuttgart)

pe, den Saalbau, den Küchenbau und den Treppenturm. Die Fundierungsarbeiten für die Reittreppe wurden im Mai 1595 begonnen, für die übrigen Teile im Jahr 1596. Im Spätsommer 1597 war der gesamte weitere Bau von der Reittreppe bis zum Küchenbau im Rohbau fertig. Als letzte Rohbauarbeit wurde im Sommer 1598 das Hängewerk über dem Saalbau und der Reittreppe aufgeschlagen. Das Dach erhielten beide Bauteile im Frühjahr 1600. Die sechs Giebel müssen je- 
weils mit dem Rohbau, spätestens aber vor dem Aufschlagen des Daches versetzt worden sein.

Im September 1597 wurde mit dem Innenausbau des Küchenbaus begonnen, der kurz vorher sein Dach erhalten haben muss; 1598 wurde der Ausbau beendet. Der Innenausbau und die Inneneinrichtung des Schlosses erstreckten sich von 1600 noch bis 1605. Dies gilt insbesondere für den Rittersaal mit dem Eingangsportal, dem Prunkkamin, der Ahnentafel, den Tierskulpturen und den Deckenbildern. ${ }^{6}$ Im Jahr 1602 begannen die Vorarbeiten für eine Altane, die an die Außenfront des Rittersaals zur Hofseite hin angefügt werden sollte. Der Steinmetz Servatius Körber erhielt am 2. Januar dieses Jahres den Auftrag zur Anfertigung der hierfür benötigten Werkstücke. ${ }^{69}$ Hierzu gehörten Pfeiler, Bögen, Platten unten im Hof und auf der Altane (Gang), eine Balustrade (Gesims), ein großes Rustikaportal, zwei kleinere Portale und drei Türgewände. Von diesem allem wird gesagt, er solle es vermög der Visirung unnd verstochnen Gibs aufs schönst, sauberst und fleißigst machen. Der verstochne Gibs kann sich nur speziell auf das Gipsmodell der Altane bezogen haben, denn Stegles Modell der Gesamtanlage war aus Holz angefertigt. Ob das Gipsmodell noch von Stegle stammt, bleibt offen, denn es könnte ebensogut von den Kalkschneidern (Stuckateuren) angefertigt worden sein, die in dieser Zeit mit der künstlerischen Ausgestaltung der Räume beschäftigt waren. Noch weniger ist klar, wer die Visirungen gezeichnet hat, die in dem Vertrag mit Körber mehrfach erwähnt werden.

Am 22. Oktober 1603 wurde mit dem Maurer Jacob Kauffmann vertraglich vereinbart, dass er die Fundamente für die Altane legen sollte. ${ }^{70}$ Außerdem mussten Mauern für die Portale nachträglich eingebrochen oder Türen versetzt werden, wodurch offensichtlich durch den Einbau des großen Portals ein großes Fenster des Saals dem Umbau zum Opfer fiel. Dies spricht eher dafür, dass die Altane nicht von Anfang an eingeplant war. Da die Werkstücke sicher inzwischen angefertigt waren, wurden sie gleich nach den Fundierungsarbeiten versetzt, so dass die Altane vielleicht noch in diesem Jahr fertiggestellt wurde. Im Anschluss daran oder parallel hierzu dürfte unter dem Rittersaal der Durchgang vom Innenhof zum Schlossgarten geschaffen worden sein, wodurch die wenige Jahre vorher errichtete neue Hofstube in zwei Teile zerlegt wurde. Die eigentliche Phase des Neubaus umfasst also acht Jahre, nämlich von 1595 bis 1603.

Mit dem Küchenbau endete der Neubau des Schlosses nach Stegles Plan. Es gibt keine Anhaltspunkte dafür, warum Wolfgang diese Idee nicht weiterverfolgte. Alle weiteren Bauarbeiten waren letzten Endes nur Provisorien. So wurde der Prinzessinnenbau in den Jahren 1602 und 1603 aufgestockt, zunächst nur ein, dann um zwei weitere Stockwerke, und ausgebaut. Durch die Aufstockungen lag der Prinzessinnenbau mit seiner Traufe in gleicher Höhe mit dem Langenburger

68 Fleck (wie Anm. 19), S. 54-58.

69 HZA We 50 Bü D 6k.

70 Fleck (wie Anm. 19), S. 58 f. 
Bau, so dass der erstere in seinem Aussehen dem Neubau angeglichen wurde. Ferner wurde 1602 an der Rückseite des Beamtenbaus ein alchemisches Laboratorium errichtet. ${ }^{71}$

\section{Der „Langenburger Bau“6}

Mit dem Langenburger Bau hat es eine besondere Bewandtnis, denn es wird bis auf den heutigen Tag die Ansicht vertreten, dass er erst etwa hundert Jahre später unter Graf Siegfried durch den Baumeister Paul Platz erbaut wurde. Fleck widmete dieser Frage in seiner Dissertation einen gesonderten Exkurs und wies anhand der Quellen ausführlich und eindeutig nach, dass dies nicht der Fall ist. ${ }^{72}$ In seiner Monographie schrieb er nur den einen Satz: „Die vorhandenen Bauakten zeigen klar, daß dieser erste Bauabschnitt der Langenburger Bau war und daß somit die seitherige Annahme, er sei erst 1679-83 gebaut worden, falsch ist. "73 Er verwies aber in einer Fußnote ausdrücklich auf den ausführlichen Text in der Dissertation. Diesem Hinweis ist aber offensichtlich niemand nachgegangen, denn sonst würde man nicht noch heute die falschen Angaben im Weikersheimer Schlossführer vorfinden.

Bevor Flecks Untersuchungsergebnisse vorgestellt werden, soll auf die Ursachen und Auswirkungen dieser Fehlinterpretation eingegangen werden. Ausgangspunkt hierfür ist eine Veröffentlichung von Heuss aus dem Jahr $1937 .{ }^{74} \mathrm{Er}$ schreibt über Platz als Baumeister: „Doch ist auch der ganze Ostflügel zwischen Sonnenbau und Belfried aus einer Reihe gewichtiger Gründe mit Sicherheit auf ihn zurückzuführen." Diese Gründe sind: 1. ein Vertrag von Siegfried mit Platz vom März 1683 über einen „,neuen großen Hauptbau“, der dem Saalbau an Höhe gleich, jedoch ohne Dachgiebel sein soll, 2. die Verzahnung von Langenburger und Saalbau, 3. die Anordnung der Räume an einem breiten Flur, die erst für den Frühbarock typisch sei, 4. die Jahreszahl 1689 (richtig ist 1680) an einer Kellertür des Langenburger Baus.

Diese Version machte sich auch Freeden in den ersten Auflagen des Weikersheimer Schlossführers zu eigen. Dort kann man die folgenden Sätze lesen: „Man begnügte sich mit dem Bau des mittleren Hauptflügels; auf den Ostflügel wurde zunächst verzichtet [... $]^{675}$ und: Paul Platz hat $[\ldots]$ „,den Ostflügel des Schlosses vom Saalbau bis zum Turm herübergeführt, ebenso hoch wie den ersteren, aber ohne Giebel. ${ }^{676}$ Die ersten Auflagen des Schlossführers enthalten noch keine Bi-

71 Ebd., S. 59-62.

72 Ebd., S. 168-179.

73 Fleck (wie Anm. 20), S. 10.

74 Hermann Heuss: Hohenloher Barock und Zopf. Öhringen 1937. S. 24, 26.

75 Von Freeden (wie Anm. 51), S. 2.

76 Ebd., S. 6. 
bliographie, aber es ist als sicher anzunehmen, dass Freeden die Veröffentlichung von Heuss als Informationsquelle benutzte.

Als Merten die späteren Auflagen des Schlossführers verfasste, war er in seinen Formulierungen vorsichtiger. Er schreibt: „Der der Stadt zugekehrte Ostflügel (Langenburger Bau) 1595 zusammen mit dem Südflügel (Saaltrakt) begonnen. Wieweit der Flügel damals vollendet wurde, ob er 1634 bei der Verwüstung des Schlosses durch kaiserliche Truppen beschädigt wurde, ist bisher nicht völlig geklärt.“ Er zitiert auch zwei Inschriften über die Bauarbeiten von Paul Platz, auf die noch eingegangen werden soll, im vollen Wortlaut und bemerkt hierzu: „,[sie] beziehen sich wenn nicht auf den Neubau, so doch auf den gründlichen Wiederaufbau und Umbau dieses Flügels. " ${ }^{\text {"77 }}$ In seiner Bibliographie ist auch die Veröffentlichung von Heuss aufgeführt.

Die Bautätigkeit von Paul Platz soll jetzt näher untersucht werden. Hierzu wurden die Forschungsergebnisse von Fleck in dem erwähnten Exkurs verwertet sowie einige Originaldokumente, die auch Fleck behandelte. Unter Wolfgangs Nachfolger in Weikersheim, seinem Sohn Georg Friedrich, ruhten alle größeren Bauarbeiten am Schloss, zumal dieser im Dreißigjährigen Krieg mit der kaiserlichen Acht belegt wurde und sich um seiner Sicherheit willen nicht in Weikersheim aufhalten durfte. Als im August 1634 kaiserliche Truppen Stadt und Schloss Weikersheim plünderten, wurde vieles an Einrichtungsgegenständen geraubt oder zerschlagen, aber die Bausubstanz blieb verschont.

Eine umfangreichere Bautätigkeit am Schloss setzte erst wieder mit Wolfgangs Enkel Siegfried von Hohenlohe (1619-1684), einem Sohn seines Sohnes Kraft, ein. Nachdem er zunächst den Landesteil Neuenstein gemeinsam mit seinen Brüdern regiert hatte, erhielt er nach einer Landesteilung 1677 Weikersheim als Residenz. Siegfried hatte seit 1679 den Maurermeister Paul Platz als Baumeister in seinen Diensten, der vorher einige Jahre in Würzburg tätig gewesen war.

Die wichtigsten der von Platz ausgeführten Arbeiten betrafen die Gestaltung der Fläche vor dem Schloss zum Burggraben hin. Es wurde eine neue Brücke über den Graben zur Stadt hin angelegt, und der sechseckige, bereits existierende Turm des Torwarts neben dieser Brücke erhielt eine Welsche Haube. 1679 wurde mit dem Bau eines Kellers auf dem Gelände des späteren südlichen Marstallflügels begonnen, der zu einem Drittel aus dem Erdboden herausragte. ${ }^{78}$ Die Jahreszahl 1679 am Kellertor in der Durchfahrt des Marstallflügels stimmt mit dem Baubeginn überein.

Im Schlossinnenhof wurde der Gebäudeteil abgebrochen, der noch von der alten Burg stammte und seit Wolfgangs Zeit den Hof zerschnitt. ${ }^{79}$ Durch den Langenburger Bau wurde ein Durchgang gebrochen, wodurch das Schloss einen neuen,

77 Merten (wie Anm. 52), S. 10.

78 Siehe Abb. 6. Der Saalbau befindet sich im Süden der Schlossanlage, der Langenburger Bau und der Marstall im Osten.

79 Siehe Abb. 3. 


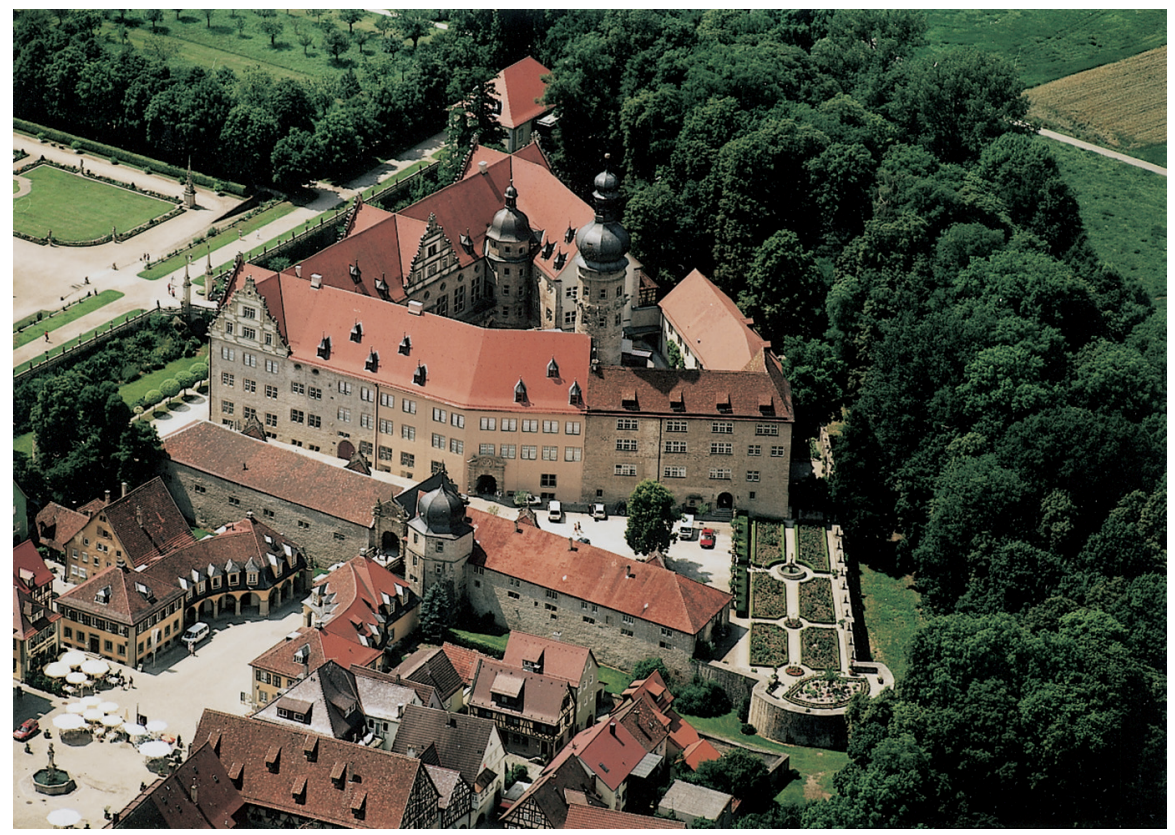

Abb. 6 Schloss Weikersheim mit Marstall, Luftbild

(Foto: Robert Schuler, Weikersheim)

repräsentativen Zugang erhielt. Der Keller des Langenburger Baus wurde durch ein Kellertor mit der Jahreszahl 1680 von der Ostseite her zugänglich gemacht und der Bergfried mit einer neuen Haube versehen.

Am 23. März 1683 wurde zwischen Siegfried von Hohenlohe und Paul Platz ein Vertrag („Accord“) über bauliche Maßnahmen geschlossen. Von diesem Vertrag existiert ein Original, versehen mit Siegfrieds und Platz' Unterschriften und zwei Siegeln, ${ }^{80}$ und eine Kopie. ${ }^{81}$ Im ersten Absatz dieses Vertrages wird ausgesagt, dass Platz seit seiner Bestallung als Baumeister vor vier Jahren schon viele Maurerarbeiten ausgeführt habe und dass jetzt wegen des noch zu führen habenden neuen großen Hauptbaus ein weiterer Vertrag aufgesetzt worden sei.

Der nächste Abschnitt beginnt mit den Worten: Nachdeme an solchem Bau das untere Stockwerck neben dem von ihme gewölbtem Keller meistentheils stehet, [...]. Dies ist der erwähnte Keller, mit dessen Bau 1679 begonnen wurde. Einige Zeilen weiter heißt es, er habe solchen Bau dergestallt aufzuführen, daß er deme Saalbau in der Höhe gleich, doch ohne Tachgiebel [sei]. 
In einem anderen Abschnitt ist von der Anfertigung von vier Portalen die Rede. Zwei davon, als Hauptportale bezeichnet, sollten die Durchfahrt zwischen dem nördlichen und südlichen Flügel des Marstalls bilden, davon eines mit dem hohenlohisch-pfälzischen Allianzwappen. Die anderen beiden Portale waren für die Durchfahrt durch den Langenburger Bau vorgesehen. Der nördliche Flügel des Marstalls muss bereits vorhanden gewesen sein, wird aber in dem Vertrag nicht ausdrücklich genannt. Im folgenden Abschnitt werden die beiden zugehörigen Kreuzgewölbe und die Giebel über den Portalen erwähnt.

Zusätzliche Informationen liefert ein undatierter Anhang zu dem Vertrag, der nur von Fleck beachtet und ausgewertet wurde. ${ }^{82}$ Er betrifft die Menge der im Einzelnen benötigten Baumaterialien und beginnt mit den Worten: Specificatio oder Uberschlag des neuen Marstalls undt darauff setzenden Baus über den alten Schloßkeller [...]. Aus dem Zusammenhang geht hervor, dass es sich um einen langgestreckten, dreigeschossigen Bau handelte. Fleck berechnete aus den angegebenen Daten die Länge und Breite des geplanten Gebäudes und kam auf 94,5 x $14 \mathrm{~m}$, was mit der von ihm gemessenen Länge des südlichen Marstallflügels von 93 x 14 m recht gut übereinstimmt. Bei den Steinhauerarbeiten werden Quader für drei Ecken des Baus genannt. Die vierte Ecke bildete ein heute nicht mehr vorhandener Torturm, der zu einem Treppenturm umgebaut werden sollte.

Durch den Tod von Siegfried am 26. April 1684 kamen die Bauarbeiten zum Erliegen und wurden nur behelfsmäßig vollendet. Die vier Portale waren zu diesem Zeitpunkt angefertigt und wurden zusammen mit den Kreuzgewölben in den Durchfahrten zwischen den beiden Marstallflügeln und zum Schlossinnenhof eingebaut. Die Mauern des geplanten Hauptbaus wurden im Erdgeschoss nur noch so weit erhöht, dass sie in der Höhe dem anderen Marstallflügel gleichkamen. Der provisorische Charakter lässt sich auch an den Fenstergewänden ablesen, die zweifellos von dem Abbruch des Gebäudes im Schlossinnenhof stammten.

$\mathrm{Zu}$ den Verwirrungen um den Langenburger Bau haben zwei Inschriften in den Portalen beigetragen. ${ }^{83}$ Die eine dieser beiden Inschriften befindet sich am inneren Portal des Langenburger Baus und lautet auszugsweise: Anno 1683 ist von dem Hochgebornen Graffen und Herrn, Herrn Siegfrieden, [...] alß vormahln allhie regierend, [...] dieser Bau auffgeführt worden. Formale architektonische und sachliche Gründe sprechen dafür, dass der Text nicht für diese Stelle vorgesehen war, sondern am geplanten Neubau angebracht werden sollte, der ja nicht zustandekam.

Die zweite Inschrift ist am inneren Portal des Marstallflügels angebracht: Anno 1684 ist dieses mit sambt dem innern neuen Schloß und Thurmbau durch Herrn Paul Platz von Belfort, Baumeister in Würtzburg, gottlob glücklich vollführt

82 Ebd.

83 Merten (wie Anm. 52), S. 10 f. - Hier werden die beiden Inschriften zum ersten Mal im vollen Wortlaut zitiert, was auch in allen späteren Auflagen des Weikersheimer Schlossführers beibehalten wurde. 
worden. Dass der Marstallflügel von Platz errichtet wurde, ist zutreffend, aber seine Verdienste um das eigentliche Schloss sind stark übertrieben. Sein Dienstherr war ja gestorben, und so hatte er bei der Formulierung freie Hand.

Nach diesen ausführlichen, aber notwendigen Erörterungen zum Beweis, dass der Langenburger Bau nicht von Paul Platz errichtet wurde, sollen noch einige Hinweise auf die Existenz dieses Baus zu Wolfgangs Zeit genannt werden, die aus der Bauphase stammen. Am 22. April 1595, als der Neubau gerade begonnen hatte, schickte Wolfgang den Steinmetz Servatius Körber zum Kloster Lichtenstern bei Löwenstein zur Besichtigung des dortigen neuen Kellers, weil, so schreibt er, [...] wir einen Baue allhier zu führen vorhabends, darunder wir gerne einen guten kalten Keller gehaben wollten, unnd in solcher Höhe, darin mit gutem und notwendigem Raum ein Fass auf 15 Heilbronner Fuder haltend liegen könnte. Mit diesem geplanten Keller kann aus räumlichen Gegebenheiten nur der Keller im Langenburger Bau gemeint sein. ${ }^{84}$

Am 19. Juni dieses Jahres bat Wolfgang den Baumeister Beringer dringend darum, nach Weikersheim zu kommen, da der Neubau schon bis unter das erste Stockwerk fortgeschritten sei. Am 23. Mai 1596 wurde der Zimmermann Gunzenhäuser angemahnt, sich zum Aufschlagen des Dachstuhls nach Weikersheim zu begeben. Der Langenburger Bau war also zu diesem Zeitpunkt im Rohbau fertiggestellt und sollte sein Dach erhalten. Am 16. Juni teilte Wolfgang ihm mit, dass sein Kommen nicht mehr nötig sei, da inzwischen sein eigener Zimmermann diese Arbeit ausgeführt habe. Andernfalls hätten die Gemächer durch das lange Regenwetter Schaden genommen. Im Februar 1597 wurde, dokumentiert durch das Klaiben der Fußböden, mit dem Innenausbau des Langenburger Baus begonnen, der wohl noch im selben Jahr zu Ende ging. ${ }^{85}$

Siegfrieds Nachfolger wurde einer seiner Neffen, Graf Carl Ludwig von Hohenlohe (1674-1756). Nachdem ihm im Mai 1708 nach einer Landesteilung durchs Los Weikersheim zugefallen war, bezog er im Januar 1709 seine Residenz. Sofort ging er daran, die nicht ausgebauten Innenräume des Langenburger Baus nach seinen eigenen Vorstellungen fertigzustellen, während er mit seiner Familie inzwischen den Beamtenbau bezog. Der Weikersheimer Kanzleidirektor Georg Tobias Pistorius schrieb in seiner Biographie des Grafen hierzu: Nach dero solennen Einzug [...] haben Ihro Hochgräfliche Gnaden sogleich das hießige Schloß, und zwar den gantzen Flügel beym Eingang, so nur gestickt und geschliert geweßen, vollends verfertigen und meubliren laßen, [...]. Die Wände und Decken im ersten und zweiten Stockwerk waren also zu Wolfgangs Zeit gestickt und geklaibt, d. h. verputzt worden. ${ }^{86}$

Auf einem Deckenbild im Rittersaal, das 1601-1602 von Balthasar Katzenberger gemalt wurde, ist im Hintergrund das kurz vorher erbaute Schloss Weikers-

84 Fleck (wie Anm. 19), S. 52.

85 Ebd., S. 50-56.

86 Carla Fandrey: Schloss Weikersheim. Neuaufl. Berlin und München 2010, S. 14 ff. 
heim abgebildet. Hier ist der Langenburger Bau in derselben Weise wie der Saalbau mit drei Giebeln verziert. Dies entsprach offenbar dem Plan von Stegle, der auch für die dritte, nicht verwirklichte Seite drei Giebel vorgesehen haben muss. Wie sich an der heute noch unveränderten Dachkonstruktion nachprüfen lässt, blieb es bei dem einen, eigentlich zum Saalbau gehörenden Giebel.

\section{Weitere Arbeiten Stegles für die Grafen von Hohenlohe}

Die folgenden Ausführungen über die Mitwirkung von Stegle bei anderen Bauvorhaben der Grafen von Hohenlohe beruhen im Wesentlichen auf den Forschungsergebnissen von Fleck in seiner Dissertation ${ }^{87}$ und seiner Monographie. ${ }^{88}$ Bei den in der Dissertation angegebenen Quellen aus dem Hohenlohe-Zentralarchiv handelt es sich um die alten Signaturen. Nur in einigen wenigen Fällen soll die Originalliteratur herangezogen und ausgewertet werden.

Was die Bau- und Reparaturarbeiten an Schloss Langenburg betrifft, so sind die Quellen in Bezug auf Stegles Anteil nicht sehr reichhaltig. Nach Freeden versah Stegle 1576 den Garten am Südhang des Schlosses mit „Gängen“ und „Hütten“ nach dem Vorbild des Stuttgarter Lustgartens. ${ }^{89}$ Die Korrespondenz zwischen Wolfgang und Stegle im Hohenlohe-Zentralarchiv beginnt mit dem Jahr 1583. ${ }^{90}$ Nach Aussage von Fleck kam Stegle zwischen 1583 und 1586 öfter nach Langenburg. Er brachte Zeichnungen der Ecktürme des Stuttgarter Lusthauses und ein anderes Mal einen Auszug aus der württembergischen Bauordnung mit. Die letztere Aussage muss allerdings etwas korrigiert werden. Am 24. Juli 1585 schrieb Wolfgang an Stegle, er habe ihm jungsten alhier zugesagt, ihm einen Auszug aus der Stuttgarter Bauordnung zukommen zu lassen, der die Besoldung der Handwerker und Taglöhner betraf. ${ }^{91}$ Aus der Formulierung jungsten alhier geht hervor, dass Stegle kurz vorher in Langenburg gewesen war. Er kam nicht erneut nach Langenburg, sondern schickte am 12. August einen Brief mit den erbetenen Unterlagen. ${ }^{92}$ Außer der Gartenanlage scheint nichts Wesentliches von Stegle gebaut worden zu sein, zumal ja wegen der bevorstehenden Erbteilung alle größeren Bauarbeiten unterblieben..$^{93}$

Geringfügige Bauarbeiten waren auch für Schloss Neuenstein geplant, wo Wolfgangs Mutter Anna, Witwe von Ludwig Casimir von Hohenlohe, ihren Sitz hatte. In den Jahren zwischen 1583 und 1586 ließ sie von dem Baumeister Thomas Fendrich Pläne für einige Wirtschaftsgebäude im Vorhof des Schlosses entwer-

87 Fleck (wie Anm. 19).

88 Fleck (wie Anm. 20).

89 Von Freeden (wie Anm. 12), S. 35 (keine Quellenangabe); Fleischhauer (wie Anm. 2), S. 80.

90 HZA We 50 Bü D 6e.

91 Ebd. Bü D 1.

92 Ebd. Bü D 6c.

93 Fleck (wie Anm. 19), S. 95 f. HZA Archiv Weikersheim A X/2/2 (alte Signatur). 
fen. Zur Beurteilung wurde Stegle herangezogen. Das muss im Mai 1586 gewesen sein, wie aus einem noch zu besprechenden Gutachten zum Schlossbau in Döttingen hervorgeht. Die geplanten Wirtschaftsgebäude wurden nicht gebaut. ${ }^{94}$ Ausführlich ist über den Bau von Schloss Döttingen am Kocher zu berichten. Döttingen war Sitz eines hohenlohischen Amts. Bald nach 1580 wurde unter Wolfgang von Hohenlohe als Bauherrn mit dem Umbau der alten Wasserburg zu einem Schloss begonnen, was einem Neubau gleichkam; der Bau war Mitte 1587 beendet. Die Pläne fertigte Fendrich an, der auch die Bauaufsicht hatte. Seine Grundrisse sind erhalten geblieben und befinden sich im Hohenlohe-Zentralarchiv. Das Schloss hatte bescheidene Ausmaße, war dreigeschossig und umschloss mit drei Flügeln im Süden, Westen und Norden und einer hohen Schildmauer im Osten einen rechteckigen Hof.

In bautechnischer Hinsicht war Fendrichs Schlossbau keine Meisterleistung, denn es stellten sich gravierende Mängel heraus. Daher wurde Stegle nach Döttingen gebeten, um die entstandenen Schäden zu besichtigen. Stegle verfasste am 10. Mai 1586 ein ausführliches, sieben Seiten umfassendes Gutachten. ${ }^{95}$ Dieses Gutachten ist das einzige noch erhaltene Dokument, das ihn als einen erfahrenen Baumeister ausweist. Es sollen daher längere Textabschnitte hieraus zitiert werden. ${ }^{96}$

In der Einleitung zu seinem Gutachten schreibt Stegle an Wolfgang, dass er von ihm den Auftrag habe, das ich inn dem Herabraisen nach Neuwenstein das Haus Dettingen am Kochen besichtigen unnd darüber mein unnderthenigen Bericht unnd Bedenckhen stellen soll. Stegle sollte also auf dem Weg nach Neuenstein in Döttingen Station machen und war mit Sicherheit vorher in Langenburg, wo die Angelegenheit besprochen wurde. Die von ihm beobachteten Bauschäden fasste er in acht Punkten zusammen. Dann folgten in dem Gutachten seine Vorschläge zur Behebung dieser Schäden, soweit er nicht im ersten Teil darauf eingegangen war. Einige dieser Punkte sollen hier kurz behandelt werden.

Das Kellergewölbe hatte der Länge nach im Scheitel einen Riss, so dass der Kellerhals, d.h. der in den Keller hinunterführende überwölbte Gang, auf die Dauer keinen Bestand haben werde. Ursach ist die, der Werckmaister, welcher denn Eingang oder Tür des Kellerhalls gehauwen und versetzt hatt, ist dem nit fleyssig gewesen, das er die Bogenstück nit verclammert, auch die Eckquader nit zu Band eins umb das [andere] inn [das] rauhe Maurwerck hatt eintreffen lassen. Zu der Altane über dem Kellerhals bemerkt er mit leicht ironischem Unterton: Zudem so ist die Alltan uff dem Kellerhalls so fleyssig gemacht, das die Blatten gar hüpsch zusamengefiegt seind, dermassen, das einer einen Finger

94 Ebd., S. 82 f. HZA A X/2/10 (alte Signatur).

95 HZA We 50 Bü D 9.

96 Gelegentlich vorkommende offensichtliche Schreibfehler wurden verbessert, fehlende Wörter in eckigen Klammern ergänzt. 
zwüschen denn Fugen hineinstossen kann. Wan es regnet, so laufft das Wasser durch die Alltan unndt durch das Gewelb hindurch wie durch einen Laugensack. Die Kellerfenster waren so ungünstig angeordnet, dass der Keller im Sommer zu warm, im Winter zu kalt war. In der großen Küche verunzierte der über dem Herd angebrachte Schlot die ein Stockwerk höher gelegene Tafelstube. Stegle empfahl die Verlegung des Schlots von der Küche aus ins Freie. In den Räumen, die mit einem Kamin versehen waren, bestand Feuersgefahr, dann wo solche Cammin durch die Gebälck hinauff gefürdt seind, ist das Holtzwerck mit so liederlichen dinne Bretlein verdeckt, allso das sich zu besorgen ist, wann ettwa ein Camin ein wenig angehen sollte, so wurdt das Holtzwerck leychtlich davon angezinndt. Daher dann ein grosser Schaden entspringen möchte.

Das größte Problem waren aber die einsturzgefährdeten Mauern des Westflügels: Zum sechsten überhangen die Hauptwennd inn den dreyen Stockwerck uff dem Keller. Die unnder Wannd hanget gegen dem Hof hinein, die Wannd im andern Stockwerck hanget hinein [... ] gegen dem Bauw zuo, die Hauptwannd des dritten Stocks hanget auch gegen dem Hof hinein. Desgleichen seind auch die Böden sampt denn Zwerch-oder Schiedwenden gegen dem Hof zu dermassen gesuncken, das die Schreiner die Türbletter nit wol einrichten kennen. Sollten [sie] dan die Türbleter gar inn das Schregmas abschneyden, dardurch die Türen verderbt unnd zunichten gemacht wurden, dieweil solche Türbletter alle eingefasset seind. Ja, mann kan kein Bettladen oder Tüsch recht setzen noch stellen von wegen des, das die Böden so gar seer hangen, wie erst gemeldet. [...] Zum achten ist Not zu mercken, wie sich die Hauptmaur des Hauses gegen der Badstuben [...] weytt hinaus gethan hatt, das sie bei dem Eck, wo der Pfeyler stehet, umb aillf Zoll überhanget. Item bey dem heimlichen Gemach [...] überhanget solche Maur umb sechtzehen Zoll, zudem so hatt gemellte Maur keinen Fus oder Absatz. [...] Derwegen an dem Haus nichts erspriesslich sein kan. ${ }^{97}$ In einem kurzen Absatz zwischen der Beschreibung der Schäden und den Vorschlägen zu ihrer Behebung betont Stegle, dass alles, was er hier geschrieben habe, das ist niemand weder zu Neyd oder zur Verkleinerung geschehen, sonnder nur, wie der Augenschein laider gnugsam am Tag ist.

Was die baufälligen Mauern des Westflügels betrifft, so beschreibt er zunächst die sonst in solchen Fällen üblichen Methoden, die aber hier nichts nützen würden: Zum vierten würt droben inn dem sechsten unnd [...] achten Poncten weyttleufftig angezaigt, wie die Hauptwennd unnd Hauptmaur des Hauses uff dem Keller hangen unnd überstehen, allso das auch zu besorgen ist, mann werde solchen Wenden unnd Gebälcken mit keinem Hebgeschür zu Hillf komen [...], inn Ansehung, das sich die drey Hauptwennd (so gegen dem hofwerds seindt) eine sich herauss unnd die annder sich hinein beugt. Unnd ob mann gleych disem Bauw mit Hebgeschüren wider auffheben wollte, so würt es nit wol sein kennen, dann sollte das öberste Gebälck, uff welchem der Dachstul ist, auch 
wider inn die recht Höhe gezogen werden, wie es dan geschehen wurde, so ist es wol müglich, das die Hauptmaur bald inn dem Graben ligen würt. Inn Bedenckung, das solch Gebälck eines Thails uff der Hauptmaur ligt, welche Maur ann einem Ort sechzehen Zoll und amm anndern Ort umb aillf Zoll überhanget gegen dem Graben heraus, zudem ist sie mit dem vilfälltigen Durchbrechen ser geschwecht worden. Das einzige, was Stegle in dieser Situation empfehlen konnte, war der Abbruch dieses Flügels bis auf den Erdboden und der Wiederaufbau mit besseren Methoden:

Derhalben ist auss jetz angezaigten Ursachen mein geringfieg[ig] unnderthenig Bedencken, das solche Maur vonn dem heimlichen Gemach an zu dem vordern Eck gegen dem Dorff durchab bis uff dem Keller dem Boden gleych, wie dann das Gebauw uff dem Keller auch hinweggebrochen werden muss bis herab uff denn Boden.

Für den Neubau der Mauern gibt er detaillierte Vorschläge: Wann aber der Bauw uff dem Keller (wie erst gemeldt) abgebrochen würt, allsdann mag man vonn dem Graben nach Lenge des Kellers herauff dem Boden gleich vier Pfeyller füren, unnd wann die neuwe Maur wider anfahet auffufüren, kann mann allsdann gedachte vier Pfeyler mit sampt der neuwen Mauren eines halben Stocks hoch auffüren, damit die Maur unnd die Pfeyller frey inn einander verfasset werden. Solche Pfeyler seind der Maur zugutem geordnet, sonnder auch, das sie dem Keller zu Hillf komen, damit die Strebmauren des Kellers nit weychen mügen. Es soll inn Sonnderhait auch ein starck Eckpfeyler ann das Eck gegen der Badstuben und gegen dem Dorff gesetzt werden, damit die allt unnd die neuw Maur verfasset werdt.

Der letzte Satz bezieht sich darauf, dass der baufällige Westflügel das Mittelstück zwischen dem Süd- und Nordflügel bildete, die offenbar keine so gravierenden Bauschäden aufwiesen. Stegle machte im Anschluss an diese Ausführungen den Vorschlag, den Westflügel beim Wiederaufbau zum Hof hin zu verbreitern, das mann die Gemecher ettwas gemuttsamers darin ordnen möchte. Die Reparaturen, insbesondere der Abriss und Wiederaufbau des Westflügels, wurden dann in der Weise ausgeführt, wie es Stegle vorgeschlagen hatte. ${ }^{98}$

Stegles Gutachten ist das wichtigste Dokument aus seiner Feder, denn es zeigt seine Fähigkeit als Baumeister und lässt auch ein wenig von seiner Persönlichkeit erkennen. Er war mit der Technik des Bauens bis ins Detail wohlvertraut, kannte sich mit der Statik aus, achtete auf die praktischen Bedürfnisse und hatte Augenmaß in Bezug auf das Machbare und Notwendige. Auch der Brandschutz war ihm ein wichtiges Anliegen. Neben allen praktischen Aspekten hatte er auch Sinn für Ästhetisches, wenn er schreibt, dass die Heraufführung des Küchenschlots durch die Tafelstube der Tafelstuben ein Ungestallt geben württ und daß man beim Neubau die Räume ettwas gemuttsamers anordnen könne. Bereits in 
der „Anhaltischen Heimführung“ hatte er bei der Besichtigung der Schlösser und Lustgärten oft die Bezeichnung „,schön“, gelegentlich auch „zierlich“, „lustig“ oder „herrlich“ benutzt. Seine Beschreibung der undichten Altane ist vielleicht ein Anzeichen für einen versteckten Humor, und seine Aussage, dass er das Gutachten nicht aus Neid oder zur Herabsetzung von irgendjemand verfasst habe, könnte auf ein ausgleichendes Temperament hinweisen.

Spätestens seit diesem Gutachten wusste Wolfgang, dass er die Ausarbeitung seiner Idee einer dreieckigen Schlossanlage Stegle anvertrauen konnte. Knapp einen Monat später, nachdem Stegle am 10. Mai 1586 sein Gutachten verfasst hatte, nämlich am 3. Juni, fand der offizielle Akt der definitiven Landesteilung zwischen Wolfgang und seinen Brüdern statt, wobei er den Landesteil Weikersheim erhielt. Er wusste nun, dass er dort seine Baupläne verwirklichen konnte. Am 16. Juli wandte er sich an Robin und, da von dort keine Antwort kam, am 22. November an Stegle. Dies sei zur zeitlichen Einordnung des Gutachtens hier wiederholt.

Im Jahr 1590 begann Wolfgang mit dem Bau von Schloss Kirchberg an der Jagst, das die alte Burg ersetzen sollte. Es war als Witwensitz für Elisabeth, die Gemahlin von Wolfgangs 1590 verstorbenem Bruder Friedrich gedacht, der seit der Erbteilung auf Schloss Langenburg residiert hatte. Das Schloss liegt auf einer Bergzunge, die in das Jagsttal vorspringt. Zuerst mussten die Reste der alten Burg und einige Felspartien abgebrochen werden; anschließend wurde mit dem Bau des Südflügels und von zwei Basteien begonnen. In der Bauphase zwischen Abbruch und Neubau kam Stegle auf Initiative von Wolfgang im Juni 1591 von Stuttgart nach Kirchberg. Dort sollte er das nach den Plänen des Werkmeisters Michel Herwarth angefertigte Holzmodell des Schlosses beurteilen. Wahrscheinlich fertigte er bei diesem Aufenthalt Pläne zu den beiden, dem Südflügel vorgelagerten Basteien an. Im August war er noch einmal in Kirchberg und half beratend beim Anlegen der Basteien.

In den folgenden Jahren wurden der Südflügel und die anderen drei Flügel der rechteckigen Schlossanlage gebaut. Die Bauarbeiten dürften gegen Ende des Jahres 1596 beendet worden sein, denn aus einem Schreiben von Elisabeth von Hohenlohe im Mai 1597 geht hervor, dass sie Schloss Kirchberg seit einiger Zeit bezogen hatte. ${ }^{99}$

Unter der Regierung von Georg Friedrich von Hohenlohe-Waldenburg wurde in den Jahren 1589 bis 1594 die Stadtkirche in Waldenburg an der Stelle einer Kapelle als Vorgängerbau neu errichtet. Der Entwurf hierfür stammt von Stegle. ${ }^{100}$ Es handelte sich um eine dreischiffige Hallenkirche unter einem hohen Satteldach mit hohen Wänden und Fenstern, vier Strebepfeilern, einem polygonalen Chor im Osten und einem massiven quadratischen Turm im Westen. Dass hier

99 Fleck (wie Anm. 19), S. 88-91. HZA Archiv Weikersheim A X/2/17 (alte Signatur); Fleck (wie Anm. 20), S. $15 \mathrm{f}$.

100 HStA Stuttgart E 116, B.5 (alte Signatur). Zitiert nach Fleischhauer (wie Anm. 2), S. 81. 
bei dem Neubau einer evangelischen Kirche mit ihren Erfordernissen als Predigtkirche weitgehend auf die Formen des spätgotischen Kirchenbaus zurückgegriffen wurde, könnte man als eine Anknüpfung an die mittelalterliche Tradition interpretieren. Im Detail lassen sich aber neben gotischen Formen auch solche der Renaissance nachweisen. ${ }^{101}$

Kurz vorher, nämlich von 1588 bis 1589, wurde unter der Bauherrschaft von Agathe von Hohenlohe-Waldenburg die evangelische Pfarrkirche in Pfedelbach erbaut. Agathe war die Witwe von Eberhard von Hohenlohe und hatte ihren Sitz in Pfedelbach, während ihr Sohn Georg Friedrich in Waldenburg residierte. Die Pfarrkirche hat bis ins Detail derartig große Ähnlichkeit mit der Stadtkirche in Waldenburg, dass Fleck mit ziemlicher Sicherheit davon überzeugt war, dass auch der Plan für die Pfedelbacher Kirche von Stegle stammt, auch wenn dies nicht quellenmäßig belegt ist. ${ }^{102}$

Abschließend sollen Stegles Aufenthalte im Hohenlohischen in chronologischer Reihenfolge kurz zusammengefasst werden, soweit gesicherte Daten vorliegen. Im Frühjahr 1585 war Stegle wegen unbekannter Arbeiten in Langenburg. Im Frühjahr 1586 kam er auf Wolfgangs Bitte nach Döttingen, um die Bauschäden des Schlossneubaus zu beurteilen, und war vorher zweifellos zur Besprechung der Angelegenheit in Langenburg. Von Döttingen ging es zur Beurteilung von Bauplänen nach Neuenstein, wo er im Mai auch das Gutachten für Döttingen verfasste. Im Juni fand die definitive Landesteilung statt, wodurch Wolfgang den Landesteil Weikersheim erhielt, und im März 1587 verlegte Wolfgang seine Residenz von Langenburg nach Weikersheim.

Vom August 1588 bis März 1589 war Stegle in Weikersheim, um in Absprache mit Wolfgang Bauzeichnungen und ein Modell für den Schlossneubau anzufertigen. Wenn die Bauarbeiten für die Pfarrkirche in Pfedelbach 1588 begannen und für die Stadtkirche in Waldenburg 1589, dann müssten spätestens in diesen beiden Jahren Stegles Bauzeichnungen hierfür vorgelegen haben. Dies aber würde höchstwahrscheinlich in die Zeit von Stegles Aufenthalt in Weikersheim fallen. Es mag also sein, dass er von Weikersheim aus einen Abstecher nach Pfedelbach und Waldenburg machte, um mit der Bauherrin und dem Bauherrn das Bauvorhaben und deren spezielle Wünsche zu besprechen. Als letztes ist der Neubau von Schloss Kirchberg zu nennen; Bauherr war Wolfgang von Hohenlohe. Im Juni 1591 kam Stegle von Stuttgart nach Kirchberg und war im August noch einmal dort, beide Male in beratender Funktion. Vielleicht war er bei dieser Gelegenheit zum letzten Mal zu einem Gespräch in Weikersheim, wo er sein Modell der dreieckigen Schlossanlage vorfand, aber die Bauarbeiten noch nicht begonnen hatten.

101 Walther-Gerd Fleck: Waldenburg. Baugeschichte von Burg und Stadt (Europäische Burgen und Schlösser D 8). Braubach 2011, S. 54-66.

102 Walther-Gerd Fleck: Das Schloß Pfedelbach 1572-1988. In: WFr 77 (1993), S. 7-122, hier S. $39-42$. 